\title{
Coding for Combined Block-Symbol Error Correction
}

\author{
Ron M. Roth, Fellow, IEEE, and Pascal O. Vontobel, Senior Member, IEEE
}

\begin{abstract}
We design low-complexity error correction coding schemes for channels that introduce different types of errors and erasures: on the one hand, the proposed schemes can successfully deal with symbol errors and erasures, and, on the other hand, they can also successfully handle phased burst errors and erasures.
\end{abstract}

Index Terms-Decoding, generalized Reed-Solomon (GRS) code, Feng-Tzeng algorithm, phased burst erasure, phased burst error, symbol erasure, symbol error.

\section{INTRODUCTION}

Many data transmission and storage systems suffer from different types of errors at the same time. For example, in some data storage systems the state of a memory cell might be altered by an alpha particle that hits this memory cell. On the other hand, an entire block of memory cells might become unreliable because of hardware wear-out. Such data transmission and storage systems can be modeled by channels that introduce symbol errors and block (i.e., phased burst) errors, where block errors encompass several contiguous symbols. Moreover, if some side information is available, say based on previously observed erroneous behavior of a single or of multiple memory cells, this can be modeled as symbol erasures and block erasures.

In this paper, we design novel error correction coding schemes that can deal with both symbol and block errors and both symbol and block erasures for a setup as in Fig. 1.

- Every small square corresponds to a symbol in $F=$ $\mathrm{GF}(q)$, where $q$ is an arbitrary prime power. (In applications, $q$ is typically a small power of 2.)

- All small squares are arranged in the shape of an $m \times n$ rectangular array.

- We say that a symbol error happens if the content of a small square is altered. We say that a block error happens if one or several small squares in a column of the array are altered 1

Manuscript received February 08, 2013; revised October 31, 2013; date of current version February 26, 2014. This paper was previously presented in part at the IEEE International Symposium on Information Theory, Istanbul, Turkey, July 2013.

R. M. Roth is with the Computer Science Department, Technion-Israel Institute of Technology, Haifa 32000, Israel. This work was done in part while visiting Hewlett-Packard Laboratories, 1501 Page Mill Road, Palo Alto, CA 94304, USA (e-mail: ronny@cs.technion.ac.il).

P. O. Vontobel was with Hewlett-Packard Laboratories, 1501 Page Mill Road, Palo Alto, CA 94304, USA. He is now with the Department of Electrical Engineering, Stanford University, Stanford, CA 94305, USA, and the Department of Information Technology and Electrical Engineering, ETH Zurich, 8092 Zurich, Switzerland (e-mail: pascal.vontobel@ieee.org).

${ }^{1}$ In our setting, we think of the symbol errors and block errors as being caused by two different mechanisms. In this model, an observer cannot distinguish a block error from one or multiple symbol errors in the same column.

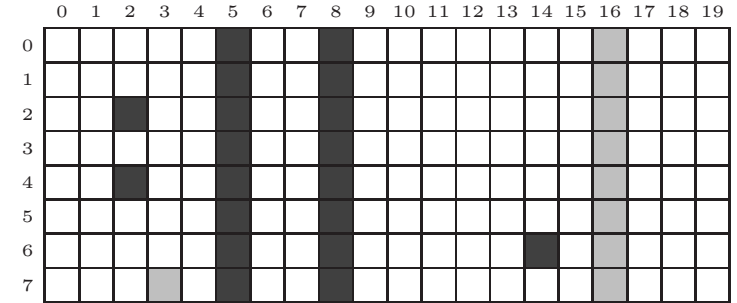

Fig. 1. Array of size $m \times n$ with symbol errors/erasures and block errors/erasures. Here, $m=8, n=20$, and there are symbol errors at positions $(2,2),(4,2)$, and $(6,14)$, a symbol erasure at position $(7,3)$, block errors in columns 5 and 8 , and a block erasure in column 16 .

- Similarly, we say that a symbol erasure happens if the content of a small square is erased and we say that a block erasure happens if all small squares in a column of the array are erased 2

We can correct such errors and erasures by imposing that the symbols in such an array constitute a codeword in some suitably chosen code $\mathbb{C}$ of length $m n$ over $F$. The two main ingredients of the code $\mathbb{C}$ that is proposed in this paper are, on the one hand, a matrix $H_{\text {in }}$ of size $m \times(m n)$ over $F$, and, on the other hand, a code $\mathcal{C}$ of length $n$ over $F$. Namely, an array forms a codeword in $\mathbb{C}$ if and only if every row of the array is a codeword in $\mathcal{C}$ once the $n$ columns have been transformed by $n$ different bijective mappings $F^{m} \rightarrow F^{m}$ derived from the matrix $H_{\text {in }}$. The resulting error-correcting coding scheme has the following salient features:

- It can be seen as a concatenated coding scheme, however with two somewhat distinctive features. First, multiple inner codes are used (one for every column encoding), and, second, all these inner codes have rate one (i.e., the encoders of these inner codes can be considered to be column scramblers).

- One can identify a range of code parameters for $\mathbb{C}$ for which (to the best of our knowledge) the resulting redundancy improves upon the best known.

- One can devise efficient decoders for combinations of symbol and block errors and erasures most relevant in practical applications. In particular, these decoders are more efficient than a corresponding decoder for a suitably chosen generalized Reed-Solomon (GRS) code of length $m n$ over $F$, assuming such a GRS code exists in the first place. (Finding efficient decoders for the general case is still an open problem.)

${ }^{2}$ The positions of the erased symbols and blocks are assumed to be provided as side information. Thus, the squares contain elements of $F$ (even at the erased positions), and some of the erased squares might in fact contain correct values. 


\section{A. Paper Overview}

The paper starts in Section $\amalg$ by considering a simplified version of the above error and erasure scenario and of the above-mentioned code construction. Namely, in this section we consider only block errors and erasures, i.e., no symbol errors or erasures. Moreover, an $m \times n$ array forms a codeword if and only if every row is a codeword in some code $\mathcal{C}$ of length $n$ (i.e., there are no bijective mappings applied to the columns); in other words, the array code considered is simply an $m$-level interleaving of $\mathcal{C}$. Our main purpose of Section is laying out some of the ideas and tools that will be used in subsequent sections; in particular, it is shown how one can take advantage of the rank of the error array in order to increase the correction capability of the array code. Nevertheless, the discussion in Section $\amalg-\mathrm{C}$ may be of independent interest in that it provides a simplified analysis of the decoding error probability of interleaved GRS codes when used in certain (probabilistic) channel models.

We then move on to Section III, which is the heart of the paper and which gives all the details of the above-mentioned code construction and compares it with other code constructions. Finally, Section IV discusses a variety of decoders for the proposed codes.

\section{B. Related Work}

The idea of exploiting the rank of the error array when decoding interleaved codes was presented by Metzner and Kapturowski in [24] and by Haslach and Vinck in [14], [15]. Therein, the code $\mathcal{C}$ is chosen to be a linear $[n, k, d]$ code over $F$, and, clearly, any combination of block errors can be corrected as long as their number does not exceed $(d-1) / 2$. In [24] and [14], it was further assumed that the set of nonzero columns in the (additive) $m \times n$ error array $E$ over $F$ is linearly independent over $F$; namely, the rank of $E$ (as a matrix over $F$ ) equals the number of block errors. It was then shown that under this additional assumption, it is possible to correct (efficiently) any pattern of up to $d-2$ block errors. Essentially, the linear independence allows to easily locate the nonzero columns in $E$, and from that point onward, the problem reduces to that of erasure decoding. A generalization to the case where the nonzero columns in $E$ are not necessarily full-rank was discussed in [15]; we will recall the latter result in mode detail in Section $\amalg-\mathrm{A}$

The case where the constituent code $\mathcal{C}$ is a GRS code has been studied in quite a few papers, primarily in the context where the contents of each block error is assumed to be uniformly drawn from $F^{m}$. In [3], Bleichenbacher et al. identified a threshold, $(m /(m+1))(d-1)$, on the number of block errors, below which the decoding failure probability approaches 0 as $d$ goes to infinity and $n / q$ goes to 0 . A better bound on the decoding error probability was obtained by Kurzweil et al. [20] and by Schmidt et al. [29], [30]. See also Brown et al. [5], Coppersmith and Sudan [6], Justesen et al. [16], Krachkovsky and Lee [19], and Wachter-Zeh et al. [34].

Turning to the main coding problem studied in this papernamely, handling combinations of symbol errors and block errors - a general solution was given by Zinov'ev [39] and Zinov'ev and Zyablov [40], using concatenated codes and their generalizations. Specifically, when using an (ordinary) concatenated code, the columns of the $m \times n$ array are set to be codewords of a linear $[m, \mathrm{k}, \mathrm{d}]$ inner code over $F$, and each of these codewords is the result of an encoding of a coordinate of an outer codeword of a second linear $[n, \mathrm{~K}, \mathrm{D}]$ code over $\operatorname{GF}\left(q^{\mathrm{k}}\right)$. It follows from the analysis in [39] and [40] that any error pattern of up to $\vartheta$ symbol errors and $\tau$ block errors can be correctly decoded, whenever

$$
2 \vartheta+1 \leq \mathrm{d}(\mathrm{D}-2 \tau) .
$$

Furthermore, such error patterns can be efficiently decoded, provided that the inner and outer codes have efficient boundeddistance error-erasure decoders.

Note that (in the nontrivial case) when $\vartheta>0$, the rate of the inner code must be (strictly) smaller than 1 . This, in turn, implies that the overall redundancy of the concatenated code has to grow (at least) linearly with $n$. A generalized concatenated (GC) code allows to circumvent this impediment. We briefly describe the approach, roughly following the formulation of Blokh and Zyablov [4]. Given a number $\tau$ of block errors and a number $\vartheta$ of symbol errors that need to be corrected, let the integer sequences

$$
\begin{gathered}
1=\mathrm{d}_{0}<\mathrm{d}_{1}<\cdots<\mathrm{d}_{v} \\
\mathrm{D}_{0} \geq \mathrm{D}_{1} \geq \cdots \geq \mathrm{D}_{v}
\end{gathered}
$$

satisfy, for every $i=0,1, \ldots, v$,

$$
2 \vartheta+1 \leq \mathrm{d}_{i}\left(\mathrm{D}_{i}-2 \tau\right)
$$

(thus, $\mathrm{D}_{0} \geq 2(\tau+\vartheta)+1$ and $\left.\mathrm{D}_{v} \geq 2 \tau+1\right)$. For $i=$ $1,2, \ldots, v$, let $\mathrm{H}_{i}$ be an $\mathrm{r}_{i} \times m$ parity-check matrix of a linear $\left[m, \mathrm{k}_{i}=m-\mathrm{r}_{i}, \mathrm{~d}_{i}\right]$ code over $F$. We further assume that these codes are (strictly) nested, so that $\mathrm{H}_{i-1}$ forms a proper $\mathrm{r}_{i-1} \times m$ sub-matrix of $\mathrm{H}_{i}$; the matrix formed by the remaining $\mathrm{r}_{i}-\mathrm{r}_{i-1}$ rows of $\mathrm{H}_{i}$ will be denoted by $\partial \mathrm{H}_{i}$ (we formally define $r_{0}=0$, along with setting $\mathrm{H}_{0}$ to be an "empty" $\mathrm{r}_{0} \times n$ matrix). Then an $m \times n$ array over $F$ is a codeword of the generalized concatenated code, if and only if the following two conditions hold:

(G1) For $i=1,2, \ldots, v$, the (partial) syndromes of the columns with respect to the partial parity-check matrix $\partial \mathrm{H}_{i}$ form a codeword of a code of length $n$ over $F^{\mathrm{r}_{i}-\mathrm{r}_{i-1}}$ with minimum distance $\mathrm{D}_{i-1}$.

(G2) The columns of the array form a codeword of a code of length $n$ over $F^{m}$ with minimum distance $\mathrm{D}_{v}$.

(Note that condition $(\mathrm{G} 2)$ could be incorporated into condition (G1) by extending the latter to $i=v+1$, with $\mathrm{H}_{v+1}$ taken as an $m \times m$ (nonsingular) parity-check matrix of the trivial code $\{\mathbf{0}\}$. Ordinary concatenated codes correspond to the case where $v=1$ and $\mathrm{D}_{0}$ is the "minimum distance" $(>n)$ of the trivial code.) It follows from [39] and [40] that the above array code construction has an efficient decoder that corrects any pattern of up to $\tau$ block errors and up to $\vartheta$ symbol errors. See also [1], [17], [27], [36], and the survey [7].

Recently, Blaum et al. [2] have proposed new erasurecorrecting codes for combined block-symbol error patterns. 
The advantage of their scheme is having the smallest possible redundancy (equaling the largest total number of symbols that can be erased) and an efficient erasure decoding algorithm. However, the parameters of their constructions are rather strongly limited: first, the array size is typically much smaller than $q$ (and, in one application, must in fact be smaller than $\log _{2} q$ ), and, secondly, verifying whether the construction actually works for given parameters becomes intractable, unless the number of block erasures or the number of symbol erasures is very small.

In [10], Gabrys et al. presented a coding scheme which is targeted mainly at applications for flash memories. In their setting, an erroneous column may have at most a prescribed number $\ell$ of symbol errors; and in addition to limiting the total number of erroneous columns, a further restriction is assumed on the number of columns with at most a prescribed number $\ell^{\prime}(<\ell)$ of symbol errors.

In Section $\coprod-C$ we will compare our coding scheme with the most relevant of the above-mentioned coding schemes.

\section{Notation}

This subsection lists the notation that will be used throughout the paper. More specialized notation will be introduced later on when needed.

For integers $a$ and $b$ with $0 \leq a<b$, we denote by $\langle a, b\rangle$ the set of integers $\{a, a+1, a+2, \ldots, b-1\}$, and $\langle b\rangle$ will be used as a shorthand notation for $\langle 0, b\rangle$. Entries of vectors will be indexed starting at 0 , and so will be the rows and columns of matrices. For a vector $\boldsymbol{u} \in F^{n}$ and a subset $W \subseteq\langle n\rangle$, we let $(\boldsymbol{u})_{W}$ be the sub-vector (in $F^{|W|}$ ) of $\boldsymbol{u}$ that is indexed by $W$. The support of $\boldsymbol{u}$ will be denoted by $\operatorname{supp}(\boldsymbol{u})$. We extend these definitions to any $m \times n$ matrix $E$ over $F$, with $(E)_{W}$ denoting the $m \times|W|$ sub-matrix of $E$ that is formed by the columns that are indexed by $W$. Column $j$ of $E$ will be denoted by $E_{j}$, and $\operatorname{supp}(E)$ will stand for the column support of $E$, namely, the set of indexes $j$ for which $E_{j} \neq \mathbf{0}$. The linear subspace of $F^{m}$ that is spanned by the columns of $E$ will be denoted by colspan $(E)$.

The ring of polynomials in the indeterminate $x$ over $F$ will be denoted by $F[x]$, and the ring of bivariate polynomials in $y$ and $x$ over $F$ will be denoted by $F[y, x]]^{3}$ For a nonzero bivariate polynomial $\varphi(y, x)=\sum_{i} \varphi_{i}(y) x^{i}$ in $F[y, x]$, we will let $\operatorname{deg}_{x} \varphi(y, x)$ stand for the $x$-degree of $\varphi(y, x)$, namely, the largest index $i$ for which $\varphi_{i}(y) \neq 0$. The $y$-degree is defined in a similar manner. The notation $F_{m, n}(y, x)$ will stand for the set of all bivariate polynomials $\varphi(y, x) \in F[y, x]$ with $\operatorname{deg}_{y} \varphi(y, x)<m$ and $\operatorname{deg}_{x} \varphi(y, x)<n$. For an element $\xi \in F$, we denote by $\mathrm{T}_{m}(y ; \xi)$ the polynomial $\sum_{i \in\langle m\rangle} \xi^{i} y^{i}$.

With any $m \times n$ matrix $E=\left(e_{h, j}\right)_{h \in\langle m\rangle, j \in\langle n\rangle}$ over $F$, we associate the bivariate polynomial

$$
E(y, x)=\sum_{h \in\langle m\rangle, j \in\langle n\rangle} e_{h, j} y^{h} x^{j}
$$

\footnotetext{
${ }^{3}$ We prefer the ordering $y, x$ over $x, y$ because the powers of $y$ and the powers of $x$ will be associated with, respectively, the rows and columns of $m \times n$ matrices like $E$.
}

in $F_{m, n}(y, x)$ (namely, the powers of $y$ index the rows and the powers of $x$ index the columns). With each column $j$ of $E$ we associate the univariate polynomial $E_{j}(y)=\sum_{h \in\langle m\rangle} e_{h, j} y^{h}$; thus, $E(y, x)=\sum_{j \in\langle n\rangle} E_{j}(y) x^{j}$.

\section{Simplified Code Construction}

In this section we consider the simplified scenario mentioned in Section I-A Namely, we consider only block errors and erasures, i.e., no symbol errors or erasures. Moreover, an $m \times n$ array forms a codeword of length $m n$ if and only if every row is a codeword in some prescribed code $\mathcal{C}$ with parameters $[n, k, d]$ (i.e., there are no bijective mappings applied to the columns); equivalently, the array code considered is simply an $m$-level interleaving of $\mathcal{C}$. If $\mathcal{C}$ is specified by an $(n-k) \times n$ parity-check matrix $H$, then the syndrome matrix $S$ is defined to be the $m \times(n-k)$ matrix $S=Y H^{\top}$, where the $m \times n$ matrix

$$
Y=\Gamma+E
$$

over $F$ represents the read out (or received) message, where the $m \times n$ matrix $\Gamma$ over $F$ represents the stored (or transmitted) codeword, and where the $m \times n$ matrix $E$ over $F$ represents the alterations that happen to $\Gamma$ over time (or during transmission). Note that our formalism treats erasures like errors, with the side information $K \subseteq\langle n\rangle$ telling us their location.

The subsections of this section are structured as follows. In Section II-A we study the error correction capabilities of the interleaved array code, where $\mathcal{C}$ is any linear $[n, k, d]$ code over $F$. Then, in Section II-B, we present an efficient decoder for the special case where $\mathcal{C}$ is a GRS code. Finally, in Section II-C we present an application of the efficient decoder of Section II-B for the probabilistic decoding of the array code under the assumption that the block errors are uniformly distributed over $F^{m}$.

\section{A. Block Errors and Erasures with Rank Constraints}

We start with Theorem 2 below that generalizes the results of [24] and [14] to the case where the set of nonzero columns of the $m \times n$ error array $E$ are not necessarily linearly independent. Note that this theorem was already stated (without proof) in the one-page abstract [15] for the error-only case (i.e., no block erasures). We include the proof of the theorem not just for the sake of completeness, but also because the proof technique will be useful in Section III as well.

Toward proving this theorem, the following lemma will be helpful.

Lemma 1 Let $\mathcal{C}$ be a linear $[n, k, d]$ code over $F$ and let $Z$ be a nonzero $m \times n$ matrix over $F$ such that each row in $Z$ is a codeword of $\mathcal{C}$. Then

$$
|\operatorname{supp}(Z)|-\operatorname{rank}(Z) \geq d-1 .
$$

Proof: Write $J=\operatorname{supp}(Z), t=|J|$, and $\mu=\operatorname{rank}(Z)$, and apply the Singleton bound to the linear $[t, \mu, \geq d]$ code over $F$ that is spanned by the rows of $(Z)_{J}$. 
Theorem 2 Let $\mathcal{C}$ be a linear $[n, k, d]$ code over $F$ and let $H$ be an $(n-k) \times n$ parity-check matrix of $\mathcal{C}$ over $F$. Fix $K$ to be a subset of $\langle n\rangle$ of size $r$. Given any $m \times(n-k)$ (syndrome) matrix $S$ over $F$, there exists at most one $m \times n$ matrix $E$ over $F$ that has the following properties:

(i) $S=E H^{\top}$, and

(ii) writing $\bar{K}=\langle n\rangle \backslash K$, the values $t=\left|\operatorname{supp}\left((E)_{\bar{K}}\right)\right|$ and $\mu=\operatorname{rank}\left((E)_{\bar{K}}\right)$ satisfy

$$
2 t+r \leq d+\mu-2 .
$$

Proof: We consider first the case where $K$ is empty. The proof is by contradiction. So, assume that $E$ and $\hat{E}$ are two distinct $m \times n$ matrices over $F$ that satisfy conditions (i)-(ii). Write

$$
\begin{aligned}
t_{\max } & =\max \{|\operatorname{supp}(E)|,|\operatorname{supp}(\hat{E})|\}, \\
\mu_{\max } & =\max \{\operatorname{rank}(E), \operatorname{rank}(\hat{E})\},
\end{aligned}
$$

and define

$$
\begin{aligned}
J & =\operatorname{supp}(E) \\
\hat{J} & =\operatorname{supp}(\hat{E}), \\
Q & =\operatorname{supp}(E) \cap \operatorname{supp}(\hat{E}) .
\end{aligned}
$$

Consider the array $Z=E-\hat{E}$. By condition (i) we get that every row in $Z$ is a codeword of $\mathcal{C}$. Now,

$$
\begin{aligned}
|\operatorname{supp}(Z)| & \leq|J|+|\hat{J}|-|Q|, \\
\operatorname{rank}(Z) & \geq \mu_{\max }-|Q|,
\end{aligned}
$$

and, so,

$$
\begin{aligned}
|\operatorname{supp}(Z)|-\operatorname{rank}(Z) & \leq|J|+|\hat{J}|-\mu_{\max } \\
& \leq 2 t_{\max }-\mu_{\max } \\
& \leq d-2,
\end{aligned}
$$

where the last inequality follows from condition (ii). Hence, by Lemma 1 we conclude that $Z=0$, namely, $E=\hat{E}$, which is a contradiction to the initial assumption.

Next, we consider the case where $r=|K|>0$. First, note that condition (ii) implies that $r \leq d+\mu-2-2 t \leq d-t-$ 2 ; in particular, every subset of $r$ columns in $H$ is linearly independent. By applying elementary linear operations to the rows of $H$, we can assume without loss of generality that the first $r$ rows of $(H)_{K}$ contain the identity matrix, whereas the remaining $n-k-r$ rows in $(H)_{K}$ are all-zero. Let $\tilde{H}$ be the $(n-k-r) \times(n-r)$ matrix which consists of the last $n-k-r$ rows of $(H)_{\bar{K}}$ : the matrix $\tilde{H}$ is a parity-check matrix of the linear $[n-r, k]$ code $\tilde{\mathcal{C}}$ over $F$ obtained by puncturing $\mathcal{C}$ on the positions that are indexed by $K$. Let $\tilde{S}$ be the $m \times(n-k-r)$ matrix which consists of the last $n-k-r$ columns of $S$. We have

$$
\tilde{S}=(E)_{\bar{K}} \tilde{H}^{\top} .
$$

Replacing (i) by $(3)$ and $\mathcal{C}$ by $\tilde{\mathcal{C}}$, we have reduced to the case where $K$ is empty. The result follows by recalling that the minimum distance of $\tilde{\mathcal{C}}$ is at least $d-r$.

The proof of Theorem 2] in [24] and [14], which was for the special case $r=0$ and $\mu=t=|\operatorname{supp}(E)|$, was carried out by introducing an efficient algorithm for decoding up to $t \leq d-2$ errors. In that algorithm, Gaussian elimination is performed on the columns of $S$, resulting in an $m \times(n-k)$ matrix $S P^{\top}$, for some invertible $(n-k) \times(n-k)$ matrix $P$ over $F$, such that the first $\mu$ columns in $S P^{\top}$ form a linearly independent set while the last $n-k-\mu$ columns in $S P^{\top}$ are all-zero. (As a matter of fact, through this Gaussian elimination, one finds the value of $\mu$.) From condition (i) in Theorem 2 we then get that

$$
S P^{\top}=E(P H)^{\top} .
$$

Let $H^{\prime}$ be the $(n-k-\mu) \times n$ matrix that is formed by the last $n-k-\mu$ rows of $P H$. It follows from (4) that the columns of $H^{\prime}$ that are indexed by $\operatorname{supp}(E)$ must be allzero. Furthermore, all the remaining columns in $H^{\prime}$ must be nonzero, or else we would have $\mu+1<d$ linearly dependent columns in $H$. It follows that $\operatorname{supp}(E)$ is the unique subset $U \subseteq\langle n\rangle$ of size $\mu$ such that $\left(H^{\prime}\right)_{U}$ is all-zero. Once the decoder identifies $\operatorname{supp}(E)$, the entries of $E$ can be found by solving linear equations. This decoding algorithm can be generalized to handle the case where $r=|K|>0$, in the spirit of the last part of the proof of Theorem 2 replace $E$, $H$, and $S$ by $(E)_{\bar{K}}, \tilde{H}$, and $\tilde{S}$, respectively.

As pointed out in [15], when $K$ is empty and the difference $t-\mu=|\operatorname{supp}(E)|-\operatorname{rank}(E)$ is assumed to be equal to some nonnegative integer $b$, then the decoding algorithm of [24] and [14] can be generalized into finding a subset $U \subseteq\langle n\rangle$ of size $t \leq(d+\mu) / 2-1$ such that colspan $\left(\left(H^{\prime}\right)_{U}\right)\left(\subseteq F^{n-k-\mu}\right)$ has dimension $b$. Letting $V$ be a subset of $U$ of size $b$ such that $\operatorname{rank}\left(\left(H^{\prime}\right)_{V}\right)=b$, the subset $W=U \backslash V$ will then point at $t-b=\mu$ columns of $E$ that form a basis of colspan $(E)$; these $\mu$ columns, in turn, are flagged as $\mu$ erasures. We will then be left with $|\operatorname{supp}(E) \backslash W|=t-\mu=b$ nonzero columns in $E$ which are yet to be located, but these can be found by applying to the received array, row by row, any boundeddistance error-erasure decoder $\mathcal{D}$ for $\mathcal{C}$. Indeed, since

$$
2 b+\mu=2 t-\mu \leq d-2,
$$

such a decoder can uniquely recover $E$ given the set $W$ of erasure locations. The extension to $r=|K|>0$ is straightforward.

Note, however, that even when we are allowed to apply the decoder $\mathcal{D}$ at no computational cost, we do not know how to find the subset $U$ efficiently as $b$ becomes large. In fact, if $b$ is large and $\mu$ is small, we may instead enumerate over the set $W$ which indexes a basis of colspan $(E)$, then use $\mathcal{D}$ to reconstruct a candidate for $E$, and finally verify that we indeed have $\operatorname{rank}(E)=\operatorname{rank}\left((E)_{W}\right)=\mu$.

\section{B. The GRS Case}

In this section, we present an efficient decoder for finding the error matrix $E$ under the conditions of Theorem 2 for the special case where $\mathcal{C}$ is a generalized Reed-Solomon (GRS) code (this decoder will then be used as a subroutine in one of the decoders to be presented in Section IV]. Specifically, hereafter in this section, we fix $\mathcal{C}$ to be an $[n, k, d=n-k+1]$ 
GRS code $\mathcal{C}_{\text {GRS }}$ over $F$ with a parity-check matrix

$$
H_{\mathrm{GRS}}=\left(\alpha_{j}^{i}\right)_{i \in\langle d-1\rangle, j \in\langle n\rangle},
$$

where $\alpha_{0}, \alpha_{1}, \ldots, \alpha_{n-1}$ are distinct nonzero elements of $F$ (without real loss of generality, and for the sake of simplicity, we restrict ourselves here to GRS codes where the column multipliers — as defined in [26, p. 148] — are all 1).

Let $E=\left(e_{h, j}\right)_{h \in\langle m\rangle, j \in\langle n\rangle}$ be an $m \times n$ (error) matrix over $F$, and let $K$ and $J$ be disjoint subsets of $\langle n\rangle$ such that

$$
J \subseteq \operatorname{supp}(E) \subseteq K \cup J
$$

(the set $J$ indexes the erroneous columns and $K$ indexes the erasure locations). For the matrix $E$, define the syndrome array as the following $m \times(d-1)$ matrix $S$ (equivalently, the bivariate polynomial $\left.S(y, x) \in F_{m, d-1}(y, x)\right)$ :

$$
S=E H_{\mathrm{GRS}}^{\top}
$$

(compare with condition (i) in Theorem 2). In addition, define the error-locator polynomial $\Lambda(x)$ and the erasure-locator polynomial $\mathrm{M}(x)$ by

$$
\begin{aligned}
& \Lambda(x)=\prod_{j \in J}\left(1-\alpha_{j} x\right), \\
& \mathrm{M}(x)=\prod_{j \in K}\left(1-\alpha_{j} x\right),
\end{aligned}
$$

respectively. Also, let the modified syndrome array be the unique $m \times(d-1)$ array $\sigma$ over $F$ (namely, the unique bivariate polynomial $\sigma(y, x)$ in $\left.F_{m, d-1}(y, x)\right)$ that satisfies the polynomial congruence

$$
\sigma(y, x) \equiv S(y, x) \mathrm{M}(x) \quad\left(\bmod x^{d-1}\right) .
$$

Finally, the (bivariate) error-evaluator polynomial $\Omega(y, x)=$ $\sum_{h \in\langle m\rangle} \Omega_{h}(x) y^{h}$ is defined by

$$
\Omega_{h}(x)=\sum_{j \in K \cup J} e_{h, j} \prod_{j^{\prime} \in(K \cup J) \backslash\{j\}}\left(1-\alpha_{j^{\prime}} x\right), \quad h \in\langle m\rangle .
$$

Lemma 3 Write $t=|J|, r=|K|$, and $\mu=\operatorname{rank}\left((E)_{J}\right)$, and suppose that

$$
2 t+r \leq d+\mu-2
$$

(see (2)). Let $\lambda(x)$ be a polynomial in $F[x]$ and $\omega(y, x)=$ $\sum_{h \in\langle m\rangle} \omega_{h}(x) y^{h}$ be a bivariate polynomial (of y-degree less than $m$ ) in $F[y, x]$ such that the following conditions are satisfied:

(P1) $\sigma(y, x) \lambda(x) \equiv \omega(y, x) \quad\left(\bmod x^{d-1}\right)$, and

(P2) $\operatorname{deg} \lambda(x) \leq(d+\mu-r) / 2-1$ and

$\operatorname{deg}_{x} \omega(y, x)<(d+\mu+r) / 2-1$.

Then there is a polynomial $u(x) \in F[x]$ such that

$$
\begin{aligned}
\lambda(x) & =\Lambda(x) u(x), \\
\omega(y, x) & =\Omega(y, x) u(x) .
\end{aligned}
$$

Proof: First, by the key equation of GRS decoding, it is known that (P1)-(P2) are satisfied for $\lambda(x)=\Lambda(x)$ and $\omega(y, x)=\Omega(y, x)$ (see, for example, [26, Section 6.3 and pp. 207-208]).
Now, let $\lambda(x)$ and $\omega(y, x)$ satisfy (P1)-(P2), fix $\ell$ to be any index in $J$, and let $U$ be a subset of $J$ of size $\mu$ such that $\ell \in U$ and $\operatorname{rank}\left((E)_{U}\right)=\mu$ (recall that $J \subseteq \operatorname{supp}(E)$ and, so, $E_{\ell} \neq 0$ ). Let $\boldsymbol{a}=\left(a_{h}\right)_{h \in\langle m\rangle}$ be a row vector in $F^{m}$ such that $(\boldsymbol{a} E)_{U}$ is nonzero on-and only on-position $\ell$.

Let $\hat{J}$ be the smallest subset of $\langle n\rangle$ such that

$$
\hat{J} \subseteq \operatorname{supp}(\boldsymbol{a} E) \subseteq K \cup \hat{J}
$$

note that $\ell \in \hat{J}$ and that $\hat{J} \subseteq\{\ell\} \cup(J \backslash U)$ and, so, $|\hat{J}| \leq$ $t-\mu+1$. Define

$$
\begin{aligned}
\hat{\Lambda}(x) & =\prod_{j \in \hat{J}}\left(1-\alpha_{j} x\right), \\
\hat{\Omega}(x) & =\frac{1}{\prod_{j^{\prime \prime} \in U \backslash\{\ell\}}\left(1-\alpha_{j^{\prime \prime}} x\right)} \cdot \sum_{h \in\langle m\rangle} a_{h} \Omega_{h}(x) \\
& =\sum_{j \in K \cup \hat{J}}\left(\boldsymbol{a} E_{j}\right) \prod_{j^{\prime} \in(K \cup \hat{J}) \backslash\{j\}}\left(1-\alpha_{j^{\prime}} x\right), \\
\hat{\sigma}(x) & =\sum_{h \in\langle m\rangle} a_{h} \sigma_{h}(x), \\
\hat{\omega}(x) & =\sum_{h \in\langle m\rangle} a_{h} \omega_{h}(x) .
\end{aligned}
$$

Observing that $\hat{\sigma}(x)$ is the modified syndrome polynomial that corresponds to the row vector $\boldsymbol{a} E$, we get from the key equation of GRS decoding that

$$
\hat{\Lambda}(x) \hat{\sigma}(x) \equiv \hat{\Omega}(x) \quad\left(\bmod x^{d-1}\right) .
$$

Moreover,

$$
\operatorname{gcd}(\hat{\Lambda}(x), \hat{\Omega}(x))=1
$$

$$
\begin{aligned}
& \operatorname{deg} \hat{\Lambda}(x)=|\hat{J}| \quad \leq t-\mu+1 \leq \frac{d-\mu-r}{2} \\
& \operatorname{deg} \hat{\Omega}(x)<|K|+|\hat{J}| \leq r+t-\mu+1 \leq \frac{d-\mu+r}{2}
\end{aligned}
$$

Multiplying both sides of (5) by $\lambda(x)$ we obtain

$$
\hat{\Lambda}(x) \lambda(x) \hat{\sigma}(x) \equiv \lambda(x) \hat{\Omega}(x) \quad\left(\bmod x^{d-1}\right) .
$$

On the other hand, by (P1) we have

$$
\lambda(x) \hat{\sigma}(x) \equiv \hat{\omega}(x) \quad\left(\bmod x^{d-1}\right),
$$

and so, combining the last two congruences, we get

$$
\hat{\Lambda}(x) \hat{\omega}(x) \equiv \lambda(x) \hat{\Omega}(x) \quad\left(\bmod x^{d-1}\right) .
$$

Now, from (P2) and (7)-(8) it follows that the degrees of the products on both sides of the last congruence are less than $d-1$. Hence, this congruence is actually a polynomial equality:

$$
\hat{\Lambda}(x) \hat{\omega}(x)=\lambda(x) \hat{\Omega}(x) .
$$

Thus, from (6) we get that $\lambda(x)$ is divisible by $\hat{\Lambda}(x)$; in particular, $\lambda(x)$ is divisible by $1-\alpha_{\ell} x$. Ranging now over all $\ell$ in $J$, we conclude that $\lambda(x)$ can be written as $\Lambda(x) u(x)$ for some polynomial $u(x) \in F[x]$. 
Finally, from (P1) and the key equation we obtain

$$
\begin{aligned}
\omega(y, x) & \equiv \sigma(y, x) \lambda(x) \\
& \equiv \sigma(y, x) \Lambda(x) u(x) \\
& \equiv \Omega(y, x) u(x) \quad\left(\bmod x^{d-1}\right),
\end{aligned}
$$

from which the equality $\omega(y, x)=\Omega(y, x) u(x)$ follows again by computing degrees: from $(\mathrm{P} 2)$ we get

$$
\operatorname{deg}_{x} \omega(y, x)<\frac{d+\mu+r}{2}-1 \leq d-1
$$

and

$$
\begin{aligned}
\operatorname{deg}_{x}( & \Omega(y, x) u(x)) \\
& =\operatorname{deg}_{x} \Omega(y, x)+(\operatorname{deg} \lambda(x)-\operatorname{deg} \Lambda(x)) \\
& <\operatorname{deg} \lambda(x)+r \\
& \leq \frac{d+\mu+r}{2}-1 \\
& \leq d-1 .
\end{aligned}
$$

This completes the proof.

Fix $J$ and $K$ to be disjoint subsets of $\langle n\rangle$, write $t=|J|$ and $r=|K|$, and let $E$ be an $m \times n$ matrix of rank $\mu$ over $F$ such that $J \subseteq \operatorname{supp}(E) \subseteq K \cup J$. Define

$$
S^{(J)}=(E)_{J}\left(\left(H_{\mathrm{GRS}}\right)_{J}\right)^{\top}
$$

and

$$
S^{(K)}=(E)_{K}\left(\left(H_{\mathrm{GRS}}\right)_{K}\right)^{\top} .
$$

Clearly, the syndrome array $S$ that corresponds to $E$ can be decomposed into

$$
S=S^{(J)}+S^{(K)},
$$

and after multiplying the respective bivariate polynomials by the erasure-locator polynomial $\mathrm{M}(x)=\prod_{j \in K}\left(1-\alpha_{j} x\right)$, we get

$$
\begin{aligned}
\sigma(y, x) & \equiv S(y, x) \mathrm{M}(x) \\
& \equiv S^{(J)}(y, x) \mathrm{M}(x)+S^{(K)}(y, x) \mathrm{M}(x)\left(\bmod x^{d-1}\right) .
\end{aligned}
$$

Now, recall (from the key equation) that the coefficients of $x^{r}, x^{r+1}, \ldots, x^{d-2}$ in $S^{(K)}(y, x) \mathrm{M}(x)$ are all zero, which means that the respective coefficients in $\sigma(y, x)$ are equal to those in $S^{(J)}(y, x) \mathrm{M}(x)$.

Let $\tilde{S}$ denote the $m \times(d-1-r)$ matrix

$$
\tilde{S}=\left(\sigma_{h, i}\right)_{h \in\langle m\rangle, i \in\langle r, d-1\rangle} .
$$

It follows from (9)-11) that

$$
\tilde{S}=(E)_{J}\left(\left(A_{\mathrm{M}} H_{\mathrm{GRS}}\right)_{J}\right)^{\top},
$$

where $A_{\mathrm{M}}$ is a $(d-1-r) \times(d-1)$ matrix over $F$ whose first row consists of the $r+1$ coefficients of $\mathrm{M}(x)$ in decreasing order (padded with $d-2-r$ zero entries), and each subsequent row is obtained from its predecessor by a shift one position to the right (compare (12) with (3)). Hence, any column indexed by $j \in J$ in the $(d-1-r) \times t$ matrix $\left(A_{\mathrm{M}} H_{\mathrm{GRS}}\right)_{J}$ takes the form

$$
\alpha_{j}^{r} \cdot \mathrm{M}\left(\alpha_{j}^{-1}\right) \cdot\left(1 \alpha_{j} \alpha_{j}^{2} \ldots \alpha_{j}^{d-2-r}\right)^{\top}
$$

\section{Input:}

- Array $Y$ of size $m \times n$ over $F$.

- Set $K$ of size $r$ of indexes of column erasures.

Steps:

1) Compute the $m \times(d-1)$ syndrome array

$$
S=Y H_{\mathrm{GRS}}^{\top} .
$$

2) Compute the modified syndrome array to be the unique $m \times$ $(d-1)$ matrix $\sigma$ that satisfies the congruence:

$$
\sigma(y, x) \equiv S(y, x) \mathrm{M}(x) \quad\left(\bmod x^{d-1}\right),
$$

where

$$
\mathrm{M}(x)=\prod_{j \in K}\left(1-\alpha_{j} x\right) .
$$

Let $\mu$ be the rank of the $m \times(d-1-r)$ matrix $\tilde{S}$ formed by the columns of $\sigma$ that are indexed by $\langle r, d-1\rangle$.

3) Using the Feng-Tzeng algorithm, compute a polynomial $\lambda(x)$ of (smallest) degree $\Delta \leq(d+\mu-r) / 2-1$ such that the following congruence is satisfied for some polynomial $\omega(y, x)$ with $\operatorname{deg}_{x} \omega(y, x)<r+\Delta$ :

$$
\sigma(y, x) \lambda(x) \equiv \omega(y, x) \quad\left(\bmod x^{d-1}\right) .
$$

If no such $\lambda(x)$ exists or the computed $\lambda(x)$ does not divide $\prod_{j \in\langle n\rangle}\left(1-\alpha_{j} x\right)$ then declare decoding failure and Stop.

4) Compute the $m \times n$ error array $E$ by the following variant of Forney's formula for error values [26 p. 195]:

$$
E_{j}(y)=\left\{\begin{array}{ll}
\frac{-\alpha_{j} \cdot \omega\left(y, \alpha_{j}^{-1}\right)}{\lambda^{\prime}\left(\alpha_{j}^{-1}\right) \cdot \mathrm{M}\left(\alpha_{j}^{-1}\right)} & \text { if } \lambda\left(\alpha_{j}^{-1}\right)=0 \\
\frac{-\alpha_{j} \cdot \omega\left(y, \alpha_{j}^{-1}\right)}{\lambda\left(\alpha_{j}^{-1}\right) \cdot \mathrm{M}^{\prime}\left(\alpha_{j}^{-1}\right)} & \text { if } j \in K \\
0 & \text { otherwise }
\end{array},\right.
$$

where $(\cdot)^{\prime}$ denotes formal differentiation.

Output:

- Decoded array $Y-E$ of size $m \times n$.

Fig. 2. Decoding of an $m$-level interleaving of a GRS code. (See Section $\llbracket-\mathrm{B}$ )

(where $\mathrm{M}\left(\alpha_{j}^{-1}\right) \neq 0$ since $K \cap J=\emptyset$ ). Hence, under the assumption that $t \leq d-1-r$ (which, in fact, holds whenever $2 t+r \leq d+\mu-2)$ we get that $\operatorname{rank}\left(\left(A_{\mathrm{M}} H_{\mathrm{GRS}}\right)_{J}\right)=t$. It now follows from (12) that

$$
\operatorname{colspan}\left((E)_{J}\right)=\operatorname{colspan}(\tilde{S})
$$

(provided that $t \leq d-1-r$ ); equivalently, for every $\boldsymbol{a} \in F^{m}$,

$$
\boldsymbol{a}(E)_{J}=\mathbf{0} \Longleftrightarrow \boldsymbol{a} \tilde{S}=\mathbf{0} .
$$

In particular, $\operatorname{rank}\left((E)_{J}\right)=\operatorname{rank}(\tilde{S})$. In fact, every $m \times s$ sub-matrix of $\tilde{S}$ which consists of $s \geq t$ consecutive columns of $\tilde{S}$ has the same rank as $(E)_{J}$.

Given $\sigma(y, x)=\sum_{h \in\langle m\rangle} \sigma_{h}(x) y^{h}$ and the number of erasures $r$, we can use the Feng-Tzeng algorithm [9] (see also the related algorithms in [8], [35], [31], [38]) to find efficiently a polynomial $\lambda(x)=\sum_{i=0}^{\Delta} \lambda_{i} x^{i}$ in $F[x]$ of smallest degree $\Delta$ such that $(\mathrm{P} 1)$ is satisfied for some $\omega(y, x)=$ $\sum_{h \in\langle m\rangle} \omega_{h}(x) y^{h}$ where $\operatorname{deg}_{x} \omega(y, x)<r+\Delta$. In other words, for every $h \in\langle m\rangle$, the sequence $\left(\sigma_{h, i}\right)_{i \in\langle r, d-1\rangle}$ satisfies the 
linear recurrence

$$
\sum_{i=0}^{\Delta} \lambda_{i} \sigma_{h, j-i}=0, \quad r+\Delta \leq j \leq d-2 .
$$

Under the assumption that $2 t+r \leq d+\mu-2$, we get from Lemma 3 that the polynomial $\lambda(x)$ found equals the errorlocator polynomial $\Lambda(x)$ (up to a normalization by a constant). From the roots of $\lambda(x)$ one can then recover the set $J$.

The decoding algorithm is summarized in Fig. 2. Next, we analyze its complexity. The syndrome computation step (Step 1 in the figure) can be carried out using $O(d m n)$ operations in $F$. Step 2 requires $O(d r m)=O\left(d^{2} m\right)$ operations to compute $\sigma(y, x)$ and $O(d m \cdot \min (d, m))$ operations to compute the rank $\mu$. The application of the Feng-Tzeng algorithm in Step 3 requires $O\left(d^{2} m\right)$ operations, and, finally, Step 4 requires $O(d n)$ operations for the Chien search and $O\left(d^{2} m\right)$ operations for computing the nonzero columns of $E$. Overall, the decoding complexity amounts to $O(d m n)$ operations for syndrome computation, $O(d n)$ for the Chien search, and $O\left(d^{2} m\right)$ for the remaining steps.

We note that Lemma 3 and the decoding algorithm in Fig. 2 apply also to the more general class of alternant codes over $F$, with $d$ now standing for the designed minimum distance of the code. Specifically, we apply the lemma and the decoding algorithm to the underlying GRS code over the appropriate extension field of $F$ : that GRS code has minimum distance $d$ and contains the alternant code as a sub-field sub-code.

\section{Application to Probabilistic Decoding}

We next provide an application of the efficient decoding algorithm of Fig. 2 for the following channel model: an $m \times n$ transmitted array over $F$ is subject to at most a prescribed number $t$ of block errors (and no erasures), such that the value (i.e., contents) of the block error in each affected column is uniformly distributed over $F^{m}$, independently of the other block-error values. (Note that in this formulation of the channel, there is a probability of $1 / q^{m}$ that an affected column will in fact be error-free; we have elected to define the channel this way in order to simplify the analysis.)

We consider the decoding problem given that the $m \times n$ transmitted array belongs to the code described in Section II-B namely, an $m$-level interleaving of an $[n, k, d=n-k+1]$ GRS code over $F$. Let $J$ be the index set of the columns that were affected (possibly by an error-free block error), where $|J| \leq t$, and let $\mu$ be the rank of the error array $E$. The decoder of Fig. 2 will fail to decode (or will decode incorrectly) only when the inequality in Lemma 3 is violated, namely, only when $\mu \leq 2|J|-d+1$. Under the assumed statistical model on the error arrays, it is easy to see that

$$
\begin{aligned}
\operatorname{Prob}\{\operatorname{rank}(E) \leq \mu\} & =\operatorname{Prob}\left\{\operatorname{rank}\left((E)_{J}\right) \leq \mu\right\} \\
& =q^{-(m-\mu)(|J|-\mu)} \cdot(1+o(1))
\end{aligned}
$$

(see [22, p. 699]), where $o(1)$ is an expression that goes to 0 as $q \rightarrow \infty$. Hence, when $m \geq d-1$, the decoding failure probability of the algorithm in Fig. 2 is bounded from above, up to a multiplicative factor $1+o(1)$, by

$$
q^{-(m+d-1-2|J|)(d-1-|J|)} \leq q^{-(m+d-1-2 t)(d-1-t)} .
$$

TABLE I

TYPES OF ERRORS AND ERASURES UNDER CONSIDERATION.

\begin{tabular}{|c||c|c|}
\hline & error & erasure \\
\hline \hline \multirow{3}{*}{ block } & $\begin{array}{c}(\mathbf{T 1}) \\
\text { column set } \mathcal{J} \\
|\mathcal{J}|=\tau\end{array}$ & $\begin{array}{c}(\mathbf{T 2}) \\
\text { column set } \mathcal{K} \\
|\mathcal{K}|=\rho\end{array}$ \\
\hline \multirow{3}{*}{ symbol } & $\begin{array}{c}(\mathbf{T 3}) \\
\text { location set } \mathcal{L} \\
|\mathcal{L}|=\vartheta\end{array}$ & $\begin{array}{c}(\mathbf{T 4}) \\
\text { location set } \mathcal{R} \\
|\mathcal{R}|=\varrho\end{array}$ \\
\hline
\end{tabular}

For $m \geq d-1$, this bound is (considerably) better than those given in [3] and [20], and is comparable to that in [29], [30] when $m$ is much larger than $d$.

\section{MAin Code Construction}

We come now to the main code construction of this paper, namely the code construction $\mathbb{C}=\left(\mathcal{C}, H_{\text {in }}\right)$ that was outlined in Section \In particular, Section Щ-A gives all the details of the channel model and the code construction, Section III-B presents the error and erasure correction capabilities of the code, and Section $\amalg$ II-C discusses a variety of examples based on specific choices for the code $\mathcal{C}$ and the matrix $H_{\mathrm{in}}$, and compares them with alternative code constructions. (Decoding algorithms for $\mathbb{C}$ will be discussed in Section [V])

\section{A. Channel Model and Code Definition}

We consider the following channel model. An $m \times n$ array $\Gamma$ over $F$ is stored (or transmitted), and $\Gamma$ is subject to the following error and erasure types (see also Table \and Fig. 1):

(T1) Block errors: a subset of columns in $\Gamma$ that are indexed by $\mathcal{J} \subseteq\langle n\rangle$ can be erroneous.

(T2) Block erasures: a subset of columns in $\Gamma$ that are indexed by $\mathcal{K} \subseteq\langle n\rangle \backslash \mathcal{J}$ can be erased.

(T3) Symbol errors: a subset of entries in $\Gamma$ that are indexed by $\mathcal{L} \subseteq\langle m\rangle \times(\langle n\rangle \backslash(\mathcal{K} \cup \mathcal{J}))$ can be erroneous.

(T4) Symbol erasures: a subset of entries in $\Gamma$ that are indexed by $\mathcal{R} \subseteq(\langle m\rangle \times(\langle n\rangle \backslash \mathcal{K})) \backslash \mathcal{L}$ can be erased.

Let the $m \times n$ matrix $\mathcal{E}$ over $F$ represent the alterations that happen to $\Gamma$ over time (or during transmission). Then the read out (or received) message is given by the $m \times n$ matrix

$$
\Upsilon=\Gamma+\mathcal{E}
$$

over $F$. Note that our formalism treats erasures like errors, with the side information $\mathcal{K}$ and $\mathcal{R}$ telling us their location. (Of course, the sets $\mathcal{J}$ and $\mathcal{L}$ are not known to the decoder $a$ priori.)

Write $\tau=|\mathcal{J}|, \rho=|\mathcal{K}|, \vartheta=|\mathcal{L}|$, and $\varrho=|\mathcal{R}|$. The total number of symbol errors (resulting from error types (T1) and (T3)) is at most $m \tau+\vartheta$ and the total number of symbol erasures (resulting from erasure types (T2) and (T4)) is $m \rho+$ $\varrho$; hence, we should be able to correct all error and erasure types (T1)-(T4) (when occurring simultaneously) while using a code of length $m n$ over $F$ with minimum distance at least $m(2 \tau+\rho)+2 \vartheta+\varrho+1$. However, such a strategy does not take 
into account the fact that errors of type (T1)-(T2) are aligned across the $m$ rows of the $m \times n$ array $\Gamma$. The next construction is designed to take advantage of such an alignment.

Definition 4 Let $\mathcal{C}$ be a linear $[n, k, d]$ code over $F$, and let $H_{\text {in }}$ be an $m \times(m n)$ matrix over $F$ that satisfies the following two properties for some positive integer $\delta$ :

(a) Every subset of $\delta-1$ columns in $H_{\mathrm{in}}$ is linearly independent (namely, $H_{\mathrm{in}}$ is a parity-check matrix of a linear code over $F$ of length $m n$ and minimum distance at least $\delta)$, and

(b) writing

$$
H_{\text {in }}=\left(\begin{array}{l|l|l|l}
H_{0} & H_{1}|\ldots| H_{n-1}
\end{array}\right),
$$

with $H_{0}, H_{1}, \ldots, H_{n-1}$ being $m \times m$ sub-matrices of $H_{\mathrm{in}}$, each $H_{j}$ is invertible over $F$.

Given $\mathcal{C}$ and $H_{\mathrm{in}}$, we define $\mathbb{C}=\left(\mathcal{C}, H_{\mathrm{in}}\right)$ to be the linear $[m n, m k]$ code over $F$ which consists of all $m \times n$ matrices

$$
\Gamma=\left(\begin{array}{l|l|l|l}
\Gamma_{0} & \Gamma_{1}|\ldots| \Gamma_{n-1}
\end{array}\right)
$$

over $F$ (where $\Gamma_{j}$ stands for column $j$ of $\Gamma$ ) such that each row in

$$
Z=\left(H_{0} \Gamma_{0}\left|H_{1} \Gamma_{1}\right| \ldots \mid H_{n-1} \Gamma_{n-1}\right)
$$

is a codeword of $\mathcal{C}$.

One can view the code $\mathbb{C}$ as a (generalized) concatenated code, where the outer code is an $m$-level interleaving of $\mathcal{C}$, such that an $m \times n$ matrix

$$
Z=\left(\begin{array}{l|l|l|l}
Z_{0} & Z_{1}|\ldots| Z_{n-1}
\end{array}\right)
$$

over $F$ is an outer codeword if and only if each row in $Z$ belongs to $\mathcal{C}$. Each column in $Z$ then undergoes encoding by an inner encoder of rate one, where the encoder of column $j$ is given by the bijective mapping $Z_{j} \mapsto H_{j}^{-1} Z_{j}$.

\section{B. Error Correction Capabilities}

This subsection discusses what combinations of errors and erasures of the types (T1)-(T4) can be handled by the code $\mathbb{C}=\left(\mathcal{C}, H_{\text {in }}\right)$ that was specified in Definition 4

Theorem 5 There exists a decoder for the code $\mathbb{C}$ that correctly recovers the transmitted array in the presence of errors of types (T1)-(T4) (which may occur simultaneously), whenever $\tau(=|\mathcal{J}|), \rho(=|\mathcal{K}|), \vartheta(=|\mathcal{L}|)$, and $\varrho(=|\mathcal{R}|)$ satisfy

$$
\begin{aligned}
& 2 \tau+\rho \leq d-2, \\
& 2 \vartheta+\varrho \leq \delta-1 .
\end{aligned}
$$

Proof: Using puncturing as in the proof of Theorem 2, it suffices to prove the theorem for the case where $\mathcal{K}$ is empty, i.e., there are no erasure events of type (T2).

The proof is by contradiction. So, assume that $\mathcal{E}$ and $\hat{\mathcal{E}}$ are two distinct $m \times n$ matrices that correspond to error events of types (T1), (T3), and (T4), with the respective sets $\mathcal{J}, \hat{\mathcal{J}}, \mathcal{L}$, and $\hat{\mathcal{L}}$ satisfying

$$
\begin{aligned}
& 2 \tau_{\max } \quad \leq d-2, \\
& 2 \vartheta_{\max }+\varrho \leq \delta-1,
\end{aligned}
$$

where

$$
\begin{aligned}
\tau_{\text {max }} & =\max \{|\mathcal{J}|,|\hat{\mathcal{J}}|\}, \\
\vartheta_{\text {max }} & =\max \{|\mathcal{L}|,|\hat{\mathcal{L}}|\},
\end{aligned}
$$

and $\varrho=|\mathcal{R}|$ (the symbol erasure set $\mathcal{R}$ is the same for both $\mathcal{E}$ and $\hat{\mathcal{E}}$ ). We will have reached a contradiction once we have shown that $\mathcal{E}-\hat{\mathcal{E}}$ is a codeword of $\mathbb{C}$ if only if $\mathcal{E}=\hat{\mathcal{E}}$.

Let $Z$ be the $m \times n$ matrix

$$
Z=\left(H_{0}\left(\mathcal{E}_{0}-\hat{\mathcal{E}}_{0}\right)\left|H_{1}\left(\mathcal{E}_{1}-\hat{\mathcal{E}}_{1}\right)\right| \ldots \mid H_{n-1}\left(\mathcal{E}_{n-1}-\hat{\mathcal{E}}_{n-1}\right)\right) .
$$

Observe that since the matrices $H_{0}, H_{1}, \ldots, H_{n-1}$ are all invertible over $F$, we get that a column in $Z$ is zero if and only if it is zero in $\mathcal{E}-\hat{\mathcal{E}}$; in particular, $Z=0$ if and only if $\mathcal{E}=\hat{\mathcal{E}}$. Write

$$
\mathcal{Q}=\operatorname{supp}(\mathcal{E}-\hat{\mathcal{E}}) \backslash(\mathcal{J} \cup \hat{\mathcal{J}}),
$$

namely, the set $\mathcal{Q}$ indexes the columns of $\mathcal{E}-\hat{\mathcal{E}}$ that contain errors of type (T3) and (possibly part of) the erasures of type (T4). For each $j \in \mathcal{Q}$, denote by $w_{j}$ the number of nonzero entries in $\mathcal{E}_{j}-\hat{\mathcal{E}}_{j}$ (that is, $w_{j}=\left|\operatorname{supp}\left(\left(\mathcal{E}_{j}-\hat{\mathcal{E}}_{j}\right)^{\mathrm{T}}\right)\right|$ ). The total number of nonzero entries in $(\mathcal{E}-\hat{\mathcal{E}})_{\mathcal{Q}}$ satisfies:

$$
\sum_{j \in \mathcal{Q}} w_{j} \leq|\mathcal{L} \cup \hat{\mathcal{L}} \cup \mathcal{R}| \leq 2 \vartheta_{\max }+\varrho \leq \delta-1 .
$$

Consider the respective columns in $Z$ :

$$
Z_{j}=H_{j}\left(\mathcal{E}_{j}-\hat{\mathcal{E}}_{j}\right), \quad j \in \mathcal{Q} .
$$

Each $Z_{j}$ is a nontrivial linear combination of $w_{j}$ columns of $H_{\text {in }}$, and, obviously, for distinct indexes $j$ these combinations involve disjoint sets of columns of $H_{\text {in }}$. Recalling that every subset of $\sum_{j \in \mathcal{Q}} w_{j}(\leq \delta-1)$ columns in $H_{\text {in }}$ is linearly independent, we thus get that the set of columns of $(Z)_{\mathcal{Q}}$ is linearly independent, that is,

$$
\operatorname{rank}(Z) \geq \operatorname{rank}\left((Z)_{\mathcal{Q}}\right)=|\mathcal{Q}| .
$$

On the other hand,

$$
|\operatorname{supp}(Z)| \leq|\mathcal{J}|+|\hat{\mathcal{J}}|+|\mathcal{Q}| \leq 2 \tau_{\max }+|\mathcal{Q}| \leq d-2+|\mathcal{Q}|
$$

and, so,

$$
|\operatorname{supp}(Z)|-\operatorname{rank}(Z) \leq d-2 .
$$

Hence, we conclude from Lemma1 that each row in $Z$ belongs to $\mathcal{C}$ only if $Z=0$. Equivalently, $\mathcal{E}-\hat{\mathcal{E}}$ belongs to $\mathbb{C}$ if only if $\mathcal{E}=\hat{\mathcal{E}}$, as promised.

Remark 6 We draw the attention of the reader to the condition on $\tau$ and $\rho$ in Theorem [5, namely, that the expression $2 \tau+\rho$ be at most $d-2$, rather than the (more common) requirement that it be at most $d-1$. It is this slightly stronger condition that, implicitly, provides the required redundancy for correcting the (additional) symbol errors and erasures. 
Remark 7 Theorem 5 indirectly implies a dependence of the correction capability of errors of type (T3)-(T4) on the parameter $m$, which, in turn, is part of the specification of the errors of type (T3)-(T4). Specifically, the largest possible value for $\delta$ can be the minimum distance of any linear code of length $m n$ and redundancy $m$ over $F$. Of course, one may re-arrange the array by grouping together non-overlapping sets of $s$ columns, for some integer $s>0$, to form an $m^{\prime} \times n^{\prime}$ array where $m^{\prime}=s m$ and $n^{\prime}=n / s$ (assuming the latter ratio is an integer). The block errors and the symbol errors will remain so also in this modified setting, except that the block errors will be more structured than just being phased with respect to the (new) parameter $m^{\prime}$. If this additional structure is not taken into account, the code $\mathbb{C}$ is bound to be sub-optimal. $\square$

Remark 8 In contrast to the previous remark, if $m$ is (much) larger than the redundancy needed from a linear code of length $m n$ and minimum distance $\delta$ as in Theorem 5 we may partition each column in the original array into $s$ new columns, thereby forming an $m^{\prime} \times n^{\prime}$ array, where $m^{\prime}=m / s$ and $n^{\prime}=s n$, such that $m^{\prime}$ is (just) the redundancy required from a linear code of length $m n$ and minimum distance $\delta$. Of course, this will increase the number of block errors by a factor of $s$, yet as long as $n^{\prime}$ is sufficiently small to allow us to use a maximum-distance separable (MDS) code for $\mathcal{C}$ (as will be the case in the examples in Section [II-C), we will obtain a gain in the redundancy: it will reduce from $(2 \tau+\rho) m+m$ to $(2 \tau+\rho) m+m^{\prime}$. Thus, the code $\mathbb{C}$ is suitable for (the rather practical) scenarios where the number of symbol errors is relatively small compared to the block-error length $m$.

Remark 9 One may speculate whether Theorem 5 holds for the following more general definition of $\mathbb{C}$ : instead of requiring that each row of $Z$ in (14) be a codeword of $\mathcal{C}$, require that $Z$ belong to a linear $[n, k, d]$ code over $\operatorname{GF}\left(q^{m}\right)$, where each column of $Z$ is regarded as an element of the latter field with respect to some basis of that field over $F$. It turns out that Theorem 5 does not hold for this more general setting, as shown by the following counterexample.

Let $d=2$ (i.e., $\tau=\rho=0$ ). Assume that $n \leq q^{m}-1$ and let $C_{0}, C_{1}, C_{2}, \ldots, C_{n-1}$ be any $n$ distinct powers of an $m \times m$ companion matrix of some irreducible polynomial of degree $m$ over $F$ [22, p. 106], where $C_{0}=I$ (the $m \times m$ identity matrix). Then

$$
\left\{Z=\left(Z_{0}\left|Z_{1}\right| \ldots \mid Z_{n-1}\right): \sum_{j \in\langle n\rangle} C_{j} Z_{j}=\mathbf{0}\right\}
$$

is a linear $[n, n-1,2]$ code over $\operatorname{GF}\left(q^{m}\right)$ (with the columns of each $Z$ being the codeword coordinates). The respective code $\mathbb{C}$ would then be written as

$$
\left\{\Gamma=\left(\Gamma_{0}\left|\Gamma_{1}\right| \ldots \mid \Gamma_{n-1}\right): \sum_{j \in\langle n\rangle} C_{j} H_{j} \Gamma_{j}=\mathbf{0}\right\} \text {. }
$$

If $\delta \geq 3$ then the $2 m$ columns of $H_{0}$ and $H_{1}$ are all nonzero and distinct. Therefore, we can select $C_{1} \neq I$ so that the first column (say) in $C_{1} H_{1}$ equals the first column (say) in $H_{0}=C_{0} H_{0}$. Yet this means that there exists a nonzero $\Gamma \in \mathbb{C}$ that contains only two nonzero entries. Therefore, $\mathbb{C}$ cannot have a decoder that corrects all one-symbol error patterns.

\section{Examples}

In this subsection, we consider various special choices for $\mathcal{C}$ and $H_{\text {in }}$ and demonstrate the properties of the resulting code $\mathbb{C}=\left(\mathcal{C}, H_{\text {in }}\right)$. Specifically, in Example 10 below, we discuss complexity advantages, in an error-detection setting, that the construction $\mathbb{C}$ can offer (with a suitable choice for $\mathcal{C}$ and $H_{\text {in }}$ ) over MDS codes with the same length and size. In Examples 11 and 12, we show cases where $\mathbb{C}$ is MDS (in fact, GRS), and, in contrast, we exhibit in Example 13 a choice of parameters for which no MDS code can have the same length, size, and symbol-block correction capability as $\mathbb{C}$. In Examples 14 16, we demonstrate the advantages of the code $\mathbb{C}$ over other existing alternatives for handling errors of types (T1)-(T4), such as concatenated codes and generalized concatenated (GC) codes.

Example 10 We consider first the special case where $m n \leq$ $q+1$. Here, we can take $\mathcal{C}$ to be an MDS code over $F$ and $H_{\text {in }}$ to be a parity-check matrix of an MDS code over $F$. Under such circumstances we have $d=n-k+1$ and $\delta=m+1$, which means that it suffices that the sizes $\tau, \rho, \vartheta$, and $\varrho$ satisfy

$$
\begin{aligned}
& 2 \tau+\rho \leq n-k-1, \\
& 2 \vartheta+\varrho \leq m .
\end{aligned}
$$

The redundancy of $\mathbb{C}$, being $m(n-k)$, is then the smallest possible for this correction capability: since the total number of symbol errors can be as large as $m \tau+\vartheta$ and the total number of symbol erasures is $m \rho+\varrho$, by the Reiger bound ([21], [25]) we need a redundancy of at least $m(2 \tau+\rho)+2 \vartheta+\varrho$ symbols over $F$ in order to be able to correct all error types (T1)-(T4). Admittedly, the same performance of correction capability versus redundancy can be achieved also by a single linear $[m n, m k]$ MDS code $C$ over $F$ (which exists under the assumption that $m n \leq q+1$ ). However, as pointed out earlier, the use of such a code $C$ does not take into account the alignment of error types (T1) and (T2) across the rows of the received $m \times n$ array. It is this alignment that allows $\mathbb{C}$ to achieve the same correction capability using a code $\mathcal{C}$, which is $m$ times shorter than $C$. While we still need for $\mathbb{C}$ the parity-check matrix $H_{\text {in }}$ of an MDS code of length $m n$, the redundancy of the latter code needs to be only $m$, rather than $m(n-k)$.

To demonstrate the savings that $\mathbb{C}$ may offer compared to $\mathrm{C}$, consider the simple problem of verifying whether a given $m \times n$ array $\Gamma$ belongs to the code (namely, detecting whether errors have occurred). When using $C$, we will regard $\Gamma$ as a vector of length $m n$ over $F$ and the checking will be carried out through multiplication by an $(m(n-k)) \times(m n)$ (systematic) parity-check matrix of $\mathrm{C}$, thereby requiring up to $2 m^{2} k(n-k)$ operations (namely, additions and multiplications) in $F$. In contrast, when using $\mathbb{C}$, we will first compute the array $Z$ as in (14) while requiring less than $2 m^{2}(n-1)$ operations in $F$ (one of the matrices $H_{j}$ can be assumed to be the identity matrix); then we will compute the syndrome 
of each row of $Z$, for which we will need up to $2 m k(n-k)$ operations in $F$.

Example 11 Suppose that $m n \leq q-1$ and select $\mathcal{C}$ to be an $[n, k, d=n-k+1]$ GRS code $\mathcal{C}_{\text {GRS }}$ over $F$ as in Section $\amalg-B$ For every $j \in\langle n\rangle$, let

$$
H_{j}=\left(\beta_{\kappa, j}^{h}\right)_{h \in\langle m\rangle, \kappa \in\langle m\rangle},
$$

such that the elements $\beta_{\kappa, j}$ are distinct and nonzero in $F$ for all $\kappa \in\langle m\rangle$ and $j \in\langle n\rangle$; the respective matrix $H_{\text {in }}=\left(H_{j}\right)_{j \in\langle n\rangle}$ is then a parity-check matrix of an $[m n, m(n-1), m+1]$ GRS code over $F$. Given any $m \times n$ matrix $\Gamma=\left(\Gamma_{\kappa, j}\right)_{\kappa \in\langle m\rangle, j \in\langle n\rangle}$ over $F$, the entries of $H_{j} \Gamma_{j}$ are given by

$$
\left(H_{j} \Gamma_{j}\right)_{h}=\sum_{\kappa \in\langle m\rangle} \Gamma_{\kappa, j} \beta_{\kappa, j}^{h}, \quad h \in\langle m\rangle .
$$

(Recall the definition of $\Gamma_{j}$ from Definition 4 4 ) Hence, $\Gamma$ is in $\mathbb{C}=\left(\mathcal{C}, H_{\text {in }}\right)$ if and only if

$$
\sum_{\kappa \in\langle m\rangle} \sum_{j \in\langle n\rangle} \Gamma_{\kappa, j} \alpha_{j}^{i} \beta_{\kappa, j}^{h}=0, \quad h \in\langle m\rangle, \quad i \in\langle d-1\rangle .
$$

(Note that if $\beta_{\kappa, j}$ depended only on $\kappa$, then $\mathbb{C}$ could be seen as a two-dimensional shortening of a two-dimensional cyclic code; see, for example [28].)

Example 12 We show that sometimes the construction $\mathbb{C}$ in Example 11 is an MDS code. Assume therein that $m$ divides $q-1$ and that for every $j \in\langle n\rangle$, the multiplicative order of $\alpha_{j}$ divides $(q-1) / m$ (thus, each $\alpha_{j}$ has $m$ distinct $m$ th roots in $F$ ). For every $j \in\langle n\rangle$, select $\beta_{0, j}, \beta_{1, j}, \ldots, \beta_{m-1, j}$ to be the distinct roots of $\alpha_{j}$ in $F$. Then $\Gamma \in \mathbb{C}$ if and only if

$$
\sum_{\kappa \in\langle m\rangle} \sum_{j \in\langle n\rangle} \Gamma_{\kappa, j} \beta_{\kappa, j}^{h+m i}=0, \quad h \in\langle m\rangle, \quad i \in\langle d-1\rangle .
$$

The latter condition, in turn, is equivalent to $\Gamma$ being a codeword of a GRS code of length $m n$ and redundancy $(d-1) m$ over $F$.

In contrast, the following example shows that sometimes $\mathbb{C}=\left(\mathcal{C}, H_{\text {in }}\right)$ is not an MDS code, even when $\mathcal{C}$ is MDS and $H_{\text {in }}$ is a parity-check matrix of an MDS code.

Example 13 Suppose that $q$ is a power of 4 and take $m=3$ and $n=(q+2) / 3$. Select $H_{\text {in }}$ to be a parity-check matrix of a $[q+2, q-1,4]$ triply-extended GRS code over $F$ [22, p. 326] and $\mathcal{C}$ to be any linear $[n, k]$ code over $F$. Thus, $\mathbb{C}$ is a linear $[q+2,3 k]$ code over $F$. It follows from the already-proved range of the MDS conjecture that $\mathbb{C}$, being longer than $q+1$, cannot be MDS when, say, $2 \leq k \leq 1+\frac{1}{6} \sqrt{q}$ [33].

In fact, Example 13 shows that there are choices of $F, n$, $m, \tau$, and $\vartheta$ for which the construction $\mathbb{C}=\left(\mathcal{C}, H_{\text {in }}\right)$ can be realized to correct any $\tau$ block errors and any $\vartheta$ symbol errors, while, on the other hand, there are no codes over $F$ of the same length and size as $\mathbb{C}$ that can correct any $2 \tau m+\vartheta$ symbol errors (Example 14 below presents a larger range of parameters where this may happen).

In Examples 14 16, we make a running assumption that $n \leq q$, in which case $\mathcal{C}$ can be taken as an MDS code, such as a (possibly extended) GRS code. For the sake of simplicity, we will consider in these examples only the block-symbol error-only case, i.e., no erasures are present.

Example 14 Given positive $\tau, \vartheta, n$, and $F=\mathrm{GF}(q)$ (such that $2 \tau+2 \leq n \leq q$ ), we take $H_{\text {in }}$ to be a parity-check matrix of a (possibly extended) shortened $\mathrm{BCH}$ code of length $\mathrm{mn}$ over $F$, where $m$ is determined by $\vartheta, n$, and $q$ to satisfy the equality

$$
m=1+\left\lceil\frac{q-1}{q} \cdot(2 \vartheta-1)\right\rceil \cdot\left\lceil\log _{q}(m n)\right\rceil
$$

(so $m n$ may be larger than $q$; see [26, p. 260]). The code $\mathcal{C}$ is taken as a (possibly extended) $[n, k, d]$ GRS code over $F$ where $d=2 \tau+2$. The overall redundancy of $\mathbb{C}=\left(\mathcal{C}, H_{\text {in }}\right)$ is then

$$
(2 \tau+1) m=2 \tau m+1+\left\lceil\frac{q-1}{q} \cdot(2 \vartheta-1)\right\rceil \cdot\left\lceil\log _{q}(m n)\right\rceil .
$$

The first term, $2 \tau m$, on the right-hand side of (15) is the smallest redundancy possible if one is to correct any $\tau$ block errors of length $m$. The remaining term therein is the redundancy (or an upper bound thereof) of a $\mathrm{BCH}$ code that corrects any $\vartheta$ symbol errors over $F$. In comparison, a shortened $\mathrm{BCH}$ code of length $m n$ over $F$ that corrects any $\tau m+\vartheta$ symbol errors may have redundancy as large as

$$
1+\left\lceil\frac{q-1}{q} \cdot(2(\tau m+\vartheta)-1)\right\rceil \cdot\left\lceil\log _{q}(m n)\right\rceil .
$$

It can be verified that the latter expression is larger than 15 when $m n>q \geq 4$.

Example 15 We compare the performance of $\mathbb{C}$ with that of a concatenated code $\mathrm{C}$ constructed from a linear $[m, \mathrm{k}, \mathrm{d}]$ inner code over $F$ and a linear $[n, \mathrm{~K}, \mathrm{D}]$ outer code over $\mathrm{GF}\left(q^{\mathrm{k}}\right)$ (where $n \leq q$ ). By the Singleton bound, we can bound the redundancy of $\mathrm{C}$ from below by

$$
\begin{aligned}
m n-\mathrm{kK} & \geq(\mathrm{D}-1) m+(\mathrm{d}-1) n-(\mathrm{D}-1)(\mathrm{d}-1) \\
& =(\mathrm{D}-1)(m+1)-n+\mathrm{d}(n-\mathrm{D}+1) .
\end{aligned}
$$

As we mentioned already in Section $[-\mathrm{B}$ any error pattern of up to $\tau$ block errors and up to $\vartheta$ symbol errors can be correctly decoded, whenever

$$
2 \vartheta+1 \leq \mathrm{d}(\mathrm{D}-2 \tau) .
$$

Since in our setting the values $\tau$ and $\vartheta$ are prescribed, we can minimize (16) over $d$ and $D$, subject to the inequality (17). Specifically, we define $\Delta=\mathrm{D}-2 \tau$ and, from (17), we can express $\mathrm{d}$ in terms of $\Delta$ as $\mathrm{d}=\lceil(2 \vartheta+1) / \Delta\rceil$, in which case (16) becomes

$$
\begin{aligned}
& \Delta(m+1)+\left\lceil\frac{2 \vartheta+1}{\Delta}\right\rceil(n-2 \tau+1-\Delta) \\
& +(2 \tau-1)(m+1)-n \\
& \geq \Delta(m+1)+\frac{(2 \vartheta+1)(n-2 \tau+1)}{\Delta} \\
& \quad-(2 \vartheta+1)-(n-2 \tau+1)+(2 \tau-1) m .
\end{aligned}
$$


The minimum of (19) over (a real) $\Delta$ is attained at

$$
\Delta_{\text {min }}=\sqrt{\frac{(2 \vartheta+1)(n-2 \tau+1)}{m+1}} .
$$

Yet we need to take into account that both $\mathrm{d}$ and $\Delta$ are positive integers.

Case 1: $n-2 \tau<\frac{m+1}{2 \vartheta+1}$. In this range (of very small $n$ ) we have $\Delta_{\min } \leq 1$ and, so, we take $\Delta=1$; namely, for this range, it is best to let the inner code handle (exclusively) the symbol errors, and then the outer code is left to correct the block errors. For this range, the expression (18) becomes

$$
2 \tau m+2 \vartheta(n-2 \tau)
$$

which is never larger than the smallest possible redundancy, $(2 \tau+1) m$, of $\mathbb{C}$. Notice, however, that when $H_{\text {in }}$ in $\mathbb{C}$ can be taken as a parity-check matrix of an MDS code, then $m=2 \vartheta$ and therefore this range is empty.

Case 2: $n-2 \tau \geq(m+1) \cdot(2 \vartheta+1)$. In this range we have $\Delta_{\min }>2 \vartheta+1$ and, so, the expression $\lceil\cdot\rceil$ (for d) in 18 equals 1; namely, for this range, it is best to regard the symbol errors as block errors. Therefore, we take $\Delta=2 \vartheta+1$ and the expression (18) becomes

$$
2(\tau+\vartheta) m
$$

which is larger than the redundancy of $\mathbb{C}$ whenever $\vartheta>0$ (assuming that $\mathcal{C}$ in $\mathbb{C}$ is an MDS code).

Case 3: $\frac{m+1}{2 \vartheta+1} \leq n-2 \tau<(m+1) \cdot(2 \vartheta+1)$. In this range, we plug the expression for $\Delta_{\text {min }}$ into 19 , resulting in the following lower bound expression on the redundancy of $\mathrm{C}$ :

$$
\begin{aligned}
& 2 \sqrt{(2 \vartheta+1)(n-2 \tau+1)(m+1)}-(2 \vartheta+1) \\
& -(n-2 \tau+1)+(2 \tau-1) m .
\end{aligned}
$$

This lower bound is greater than the redundancy of $\mathbb{C}$ whenever

$$
\begin{aligned}
4(2 \vartheta+1) & (n-2 \tau+1)(m+1) \\
& >(2 m+(2 \vartheta+1)+(n-2 \tau+1))^{2} .
\end{aligned}
$$

Since the left-hand side of 20 is a cubic expression while the right-hand side is only quadratic, the inequality (20) is expected to hold for a range of parameters of interest, e.g., when $m, \tau$, and $\vartheta$ scale linearly with $n$.

Example 16 In many cases, the redundancy of $\mathbb{C}$ is smaller even than that of the generalized concatenated (GC) code construction defined through conditions $(\mathrm{G} 1)-(\mathrm{G} 2)$ in Section $[-\mathrm{B}$ Referring to the notation therein, we first note that if $\mathrm{D}_{v}>2 \tau+1$, then the contribution of condition $(\mathrm{G} 2)$ alone to the redundancy is already at least $\left(\mathrm{D}_{v}-1\right) m \geq(2 \tau+1) m$. Hence, we assume that $\mathrm{D}_{v}=2 \tau+1$, in which case, from (1), we get that $\mathrm{r}_{v} \geq \mathrm{d}_{v}-1 \geq 2 \vartheta$. Thus, condition (G2) induces a redundancy of at least $2 \tau m$, and condition (G1) adds a redundancy of at least

$$
\sum_{i=1}^{v}\left(\mathrm{r}_{i}-\mathrm{r}_{i-1}\right)\left(\mathrm{D}_{i-1}-1-2 \tau\right) .
$$

In Appendix $\mathrm{A}$, we show that the expression 21 is bounded from below by

$$
(2 \vartheta+1) \ln \vartheta+2 \gamma \cdot \vartheta+O(1),
$$

where $\gamma$ is Euler's constant (approximately 0.5772) [11, p. 264]. Taking $\mathcal{C}$ in $\mathbb{C}$ as an MDS code, the redundancy of $\mathbb{C}$ is then smaller than that of GC codes (with the same correction capabilities) whenever (22) exceeds $m$.

\section{Decoding Algorithms for the MAin Code Construction}

We now discuss a variety of decoders for the code $\mathbb{C}=$ $\left(\mathcal{C}, H_{\text {in }}\right)$ that was specified in Definition 4 for the case where the constituent $\operatorname{code} \mathcal{C}$ is a GRS code. Section IV-A presents a polynomial-time decoding algorithm for error and erasures of types (T1)-(T4) as far as they are correctable as guaranteed by Theorem 5, and provided that the code parameters $n, d, \delta$, $\rho$, and $\varrho$ satisfy a certain inequality (see Theorem 18 below). Then, we consider the construction $\mathbb{C}$ as in Example11 (where $\mathcal{C}$ is a GRS code and $H_{\mathrm{in}}$ is a parity-check matrix of a GRS code) and present some more specialized decoders for this construction. Namely, Section IV-B discusses a decoder that handles errors and erasures of types (T1), (T2), (T4), but not of type (T3), and Section IV-C introduces a decoder that handles errors and erasures of types (T1), (T2), (T4), and some combinations of errors of type (T3), including the case where there are at most three errors of type (T3) 4 As of yet, we do not have an efficient decoder that corrects all error patterns that satisfy the conditions of Theorem 5 (even for the construction of Example 11, excepting certain special cases such as Example 12.

Assume that $m, \tau, \rho, \vartheta$, and $\varrho$ scale linearly with $n$. If $\mathbb{C}$ is replaced by a GRS code (if such a code exists) then the decoding complexity scales linearly with $\left(n^{2}\right)^{2}=n^{4}$. One of the main purposes of defining the code $\mathbb{C}=\left(\mathcal{C}, H_{\text {in }}\right)$ is the potential existence of a decoding algorithm whose complexity does not scale higher than $n^{3}$, as is the case for the special choices of $\mathbb{C}=\left(\mathcal{C}, H_{\text {in }}\right)$ and decoders in Sections IV-B and IV-C

\section{A. Polynomial-Time Decoding Algorithm}

Example 12 demonstrates one particular instance of the construction $\mathbb{C}=\left(\mathcal{C}, H_{\text {in }}\right)$ for which the decoder guaranteed by Theorem 5 has an efficient implementation (simply because in this case the code $\mathbb{C}$ is a GRS code). In this section, we exhibit a much wider range of instances for which decoding can be carried out in polynomial-time complexity.

Specifically, we consider the case where the code $\mathcal{C}$ is a GRS code over $F$ (and, so, $n \leq q-1$ ), and $H_{\text {in }}$ is an arbitrary $m \times(m n)$ matrix over $F$ that satisfies the two properties (a)(b) in Definition 4 The columns of $m \times n$ arrays will be

\footnotetext{
${ }^{4} \mathrm{~A}$ small number of errors of type (T3) is a realistic assumption for memory storage applications that use scrubbing, i.e., memory storage applications where a background task periodically inspects the memory for errors and corrects them if necessary. Such a background task helps avoid the accumulation of errors between the time that a program writes and reads a certain memory location.
} 
regarded as elements of the extension field $\mathrm{GF}\left(q^{m}\right)$ (according to some basis of $\operatorname{GF}\left(q^{m}\right)$ over $F$ ). When doing so, the matrix $Z$ in (14) can be seen as a codeword of a GRS code $\mathcal{C}^{\prime}$ over $\operatorname{GF}\left(q^{m}\right)$, where $\mathcal{C}^{\prime}$ has the same code locators $\left(\alpha_{j}\right)_{j \in\langle n\rangle}$ as $\mathcal{C}$ (this observation was used, for example, in [32], and more recently in [13]).

Let $\Gamma \in \mathbb{C}$ be the transmitted $m \times n$ array and let $\Upsilon$ be the received $m \times n$ array, possibly corrupted by $\tau$ errors of type (T1) and $\vartheta$ errors of type (T3), where $\tau \leq(d / 2)-1$ and $\vartheta \leq(\delta-1) / 2$. We first compute an $m \times n$ array

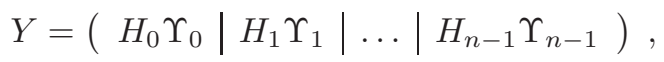

where $Y$ contains $\tau+\vartheta \leq(d+\delta-3) / 2$ erroneous columns. Regarding now $Y$ as a corrupted version of a codeword of $\mathcal{C}^{\prime}$, we can apply a list decoder for $\mathcal{C}^{\prime}$ to $Y$. Such a decoder returns a list of up to a prescribed number $L$ of codewords of $\mathcal{C}^{\prime}$, and the returned list is guaranteed to contain the correct codeword, provided that the number of erroneous columns in $Y$ does not exceed the decoding radius of $\mathcal{C}^{\prime}$. In our decoding, we will use the polynomial-time list decoder due to Guruswami and Sudan [12] (for variations of the algorithm that reduce its complexity, see [18] and [37]). For any GRS code of length $n$ and minimum distance $d$ (over any field) and for any prescribed list size $L$, their decoder will return the correct codeword as long as the number of errors does not exceed $\left\lceil n \Theta_{L}(d / n)\right\rceil-1$, where $\Theta_{L}(d / n)$ is the maximum over $s \in\{1,2, \ldots, L\}$ of the following expression:

$$
\Theta_{L, s}(d / n)=1-\frac{s+1}{2(L+1)}-\frac{L}{2 s}\left(1-\frac{d}{n}\right)
$$

(see [26, Chapter 9.5]). Thus, if $L$ is such that

$$
n \Theta_{L}(d / n) \geq(d+\delta-1) / 2
$$

then the returned list will contain the correct codeword

$$
Z=\left(H_{0} \Gamma_{0}\left|H_{1} \Gamma_{1}\right| \ldots \mid H_{n-1} \Gamma_{n-1}\right)
$$

of $\mathcal{C}^{\prime}$. For each array $Z^{\prime}$ in the list we can compute the respective array in $\mathbb{C}$,

$$
\Gamma^{\prime}=\left(\begin{array}{l}
H_{0}^{-1} Z_{0}^{\prime}\left|H_{1}^{-1} Z_{1}^{\prime}\right| \ldots \mid H_{n-1}^{-1} Z_{n-1}^{\prime}
\end{array}\right),
$$

and it follows from the proof Theorem 5 that only one such computed array $\Gamma^{\prime}$-namely, the transmitted array $\Gamma$-can correspond to an error pattern of up to $(d / 2)-1$ block errors and up to $(\delta-1) / 2$ symbol errors. Finding that array can be done simply by checking each computed $Z^{\prime}$ against the received array $\Upsilon$.

Remark 17 While the decoding scheme that we have just outlined makes essential use of an efficient list decoder for the $m$-level interleaving of $\mathcal{C}$, nothing is assumed about $H_{\text {in }}$ beyond properties (a)-(b) in Definition 4 In particular, nothing is assumed about the decoding complexity of the code over $F$ that is defined by the parity-check matrix $H_{\mathrm{in}}$.

Our decoding scheme can be generalized to handle also erasures of types (T2) and (T4) by applying a list decoder for the GRS code obtained by puncturing $\mathcal{C}^{\prime}$ on the columns that are affected by erasures: this translates into replacing $d$ by $d-\rho-\varrho$ (assuming that the latter value is positive).

The next theorem characterizes a range of parameters for which (23) holds for some polynomially-large list size $L$ (and, thus, $\mathbb{C}$ can be decoded in polynomial time).

Theorem 18 For $\mathbb{C}=\left(\mathcal{C}, H_{\text {in }}\right)$ such that $\mathcal{C}$ is a GRS code over $F$, the decoder guaranteed by Theorem 5 can be implemented by a polynomial-time algorithm, whenever

$$
d-\rho-\varrho \geq 2 \sqrt{\delta(n-\rho-\varrho)}-\delta
$$

(or, simply, whenever $d \geq 2 \sqrt{\delta n}-\delta$ in case $\rho=\varrho=0$ ).

Proof: We will assume in the proof that $\rho=\varrho=0$; the general case follows by observing that any puncturing of $\mathcal{C}^{\prime}$ on $\rho+\varrho$ positions results in a GRS code of length $n-\rho-\varrho$ and minimum distance $d-\rho-\varrho$.

Our proof will be complete once we identify a polynomially-large $L$ for which (23) holds. We take $L$ to be such that

$$
\frac{d}{n} \geq 2 \sqrt{\frac{\delta-1}{n}}-\frac{\delta-1}{n}+\frac{2}{L+1} .
$$

It readily follows from that there exists such an $L$ which is at most quadratic in $n$.

Define $s$ to be

$$
s=L-\left\lceil L \cdot \sqrt{\frac{\delta-1}{n}}\right\rceil .
$$

From 25) we have

$$
\frac{d}{n} \geq \frac{s}{L-s} \cdot \frac{\delta-1}{n}+\frac{L+1-s}{L+1},
$$

which can also be rewritten as

$$
\frac{d}{n} \geq \frac{2 s}{L-s}\left(\frac{\delta-1}{2 n}+\frac{L}{2 s}+\frac{s+1}{2(L+1)}-1\right) .
$$

Multiplying both sides by $(L-s) /(2 s)$ and rearranging terms yields

$$
1-\frac{s+1}{2(L+1)}-\frac{L}{2 s}\left(1-\frac{d}{n}\right) \geq \frac{d}{2 n}+\frac{\delta-1}{2 n},
$$

which is equivalent to

$$
\Theta_{L, s}(d / n) \geq \frac{d+\delta-1}{2 n} .
$$

This, in turn, implies 23.

The range of parameters in (24) may potentially be increased in light of a recent result of Guruswami and Xing on list decoding of interleaved GRS codes [13].

\section{B. Decoding of Errors and Erasures of Type (T1), (T2), (T4), but not of Type (T3)}

In this section, we present an efficient decoder for the code $\mathbb{C}$ when constructed as in Example 11, for the special case where $\vartheta=|\mathcal{L}|=0$ (no errors of type (T3)) 5

\footnotetext{
${ }^{5}$ This special case has also been considered in [29], yet under the setting of Section II-C namely, where the decoding algorithm may fail with a (controlled) positive probability.
} 
An $m \times n$ matrix $\Gamma$ is transmitted and an $m \times n$ matrix

$$
\Upsilon=\Gamma+\mathcal{E}
$$

is received, where $\mathcal{E}=\left(\varepsilon_{\kappa, j}\right)_{\kappa \in\langle m\rangle, j \in\langle n\rangle}$ is an $m \times n$ error matrix, with $\mathcal{J}(\subseteq\langle n\rangle)$ (respectively, $\mathcal{K}(\subseteq\langle n\rangle)$ ) indexing the columns in which block errors (respectively, block erasures) have occurred, and $\mathcal{R}(\subseteq\langle m\rangle \times\langle n\rangle)$ is a nonempty set of positions where symbol erasures have occurred 6 We assume that $d, \tau(=|\mathcal{J}|)$, and $\rho(=|\mathcal{K}|)$ satisfy

$$
2 \tau+\rho \leq d-2
$$

and that $\varrho(=|\mathcal{R}|)$ satisfies

$$
0<\varrho \leq m .
$$

Define

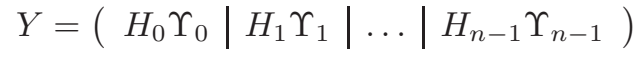

and

$$
\begin{aligned}
E=\left(e_{h, j}\right)_{h \in\langle m\rangle, j \in\langle n\rangle} & \\
& =\left(H_{0} \mathcal{E}_{0}\left|H_{1} \mathcal{E}_{1}\right| \ldots \mid H_{n-1} \mathcal{E}_{n-1}\right) .
\end{aligned}
$$

Clearly,

$$
Y=Z+E
$$

where $Z$ is given by (14). In particular, every row in $Z$ is a codeword of $\mathcal{C}_{\mathrm{GRS}}$.

Next, write $\mathcal{R}=\left\{\left(\kappa_{\ell}, j_{\ell}\right)\right\}_{\ell \in\langle\varrho\rangle}$. For each $\ell \in\langle\varrho\rangle$, define the following univariate polynomial (of degree $\varrho-1$ )

$$
\begin{aligned}
\mathrm{B}^{(\ell)}(y) & =\sum_{i \in\langle\varrho\rangle} \mathrm{B}_{i}^{(\ell)} y^{i} \\
& =\prod_{(\kappa, j) \in \mathcal{R} \backslash\left\{\left(\kappa_{\ell}, j_{\ell}\right)\right\}} \frac{1-\beta_{\kappa, j} y}{1-\beta_{\kappa, j} \beta_{\kappa_{\ell}, j \ell}^{-1}},
\end{aligned}
$$

and let $\boldsymbol{e}^{(\ell)}=\left(e_{j}^{(\ell)}\right)_{j \in\langle n\rangle}$ denote row $\varrho-1$ of the $(m+\varrho-1) \times$ $n$ matrix $\mathrm{B}^{(\ell)}(y) E(y, x)$ (where we recall the definition of $E(y, x)$ from Section [-C). We have

$$
\operatorname{supp}\left(\boldsymbol{e}^{(\ell)}\right) \subseteq \mathcal{J} \cup \mathcal{K} \cup\left\{j_{\ell}\right\}, \quad \ell \in\langle\varrho\rangle .
$$

Indeed, the contribution of a symbol erasure at position $(\kappa, j)$ in $\mathcal{E}$ to the column $E_{j}(y)$ of $E(y, x)$ is an additive term of the form

$$
\varepsilon_{\kappa, j} \cdot \mathrm{T}_{m}\left(y ; \beta_{\kappa, j}\right)=\varepsilon_{\kappa, j} \cdot \frac{1-\left(\beta_{\kappa, j} y\right)^{m}}{1-\beta_{\kappa, j} y}
$$

(where we recall the definition of $\mathrm{T}_{m}(\cdot ; \cdot)$ from Section [-C]; so, if $(\kappa, j) \neq\left(\kappa_{\ell}, j_{\ell}\right)$ then the product

$\mathrm{B}^{(\ell)}(y) \cdot \varepsilon_{\kappa, j} \cdot \frac{1-\left(\beta_{\kappa, j} y\right)^{m}}{1-\beta_{\kappa, j} y}=\varepsilon_{\kappa, j} \cdot \frac{\mathrm{B}^{(\ell)}(y)}{1-\beta_{\kappa, j} y} \cdot\left(1-\left(\beta_{\kappa, j} y\right)^{m}\right)$

is a polynomial in which the powers $y^{\varrho-1}, y^{\varrho}, \ldots, y^{m-1}$ have zero coefficients.

\footnotetext{
${ }^{6}$ When performing arithmetic operations on $\Upsilon$, we assume that the erased entries in the array are preset to some arbitrarily-selected elements of $F$, whereas the sets $\mathcal{K}$ and $\mathcal{R}$ are provided as side information. Thus, $\mathcal{E}$ is also an array over $F$.
}

Now, for each $\ell \in\langle\varrho\rangle$, every row in the $(m+\varrho-1) \times n$ array $Z^{(\ell)}(y, x)=\mathrm{B}^{(\ell)}(y) Z(y, x)$ is a codeword of $\mathcal{C}_{\mathrm{GRS}}$. Therefore, by applying a decoder for $\mathcal{C}_{\mathrm{GRS}}$ to row $\varrho-1$ of $Z^{(\ell)}$ with $\rho+1$ erasures indexed by $\mathcal{K} \cup\left\{j_{\ell}\right\}$, we should be able to decode the vector $e^{(\ell)}$, based on our assumption (26). It follows from the definition of $\boldsymbol{e}^{(\ell)}$ that for every $j \in\langle n\rangle$,

$$
\left(\begin{array}{c}
e_{j}^{(0)} \\
e_{j}^{(1)} \\
\vdots \\
e_{j}^{(\varrho-1)}
\end{array}\right)=\left(\begin{array}{cccc}
\mathrm{B}_{0}^{(0)} & \mathrm{B}_{1}^{(0)} & \ldots & \mathrm{B}_{\varrho-1}^{(0)} \\
\mathrm{B}_{0}^{(1)} & \mathrm{B}_{1}^{(1)} & \ldots & \mathrm{B}_{\varrho-1}^{(1)} \\
\vdots & \vdots & \vdots & \vdots \\
\mathrm{B}_{0}^{(\varrho-1)} & \mathrm{B}_{1}^{(\varrho-1)} & \ldots & \mathrm{B}_{\varrho-1}^{(\varrho-1)}
\end{array}\right)\left(\begin{array}{c}
e_{\varrho-1, j} \\
e_{\varrho-2, j} \\
\vdots \\
e_{0, j}
\end{array}\right) .
$$

In particular,

$$
\begin{aligned}
e_{j_{\ell}}^{(\ell)} & =\sum_{i \in\langle\varrho\rangle} B_{i}^{(\ell)} \sum_{\kappa:\left(\kappa, j_{\ell}\right) \in \mathcal{R}} \varepsilon_{\kappa, j_{\ell}} \beta_{\kappa, j_{\ell}}^{\varrho-1-i} \\
= & \sum_{\kappa:\left(\kappa, j_{\ell}\right) \in \mathcal{R}} \varepsilon_{\kappa, j_{\ell}} \beta_{\kappa, j_{\ell}}^{\varrho-1} \mathrm{~B}^{(\ell)}\left(\beta_{\kappa, j_{\ell}}^{-1}\right) \\
= & \varepsilon_{\kappa_{\ell}, j_{\ell}} \beta_{\kappa, j_{\ell}}^{\varrho-1} .
\end{aligned}
$$

Ranging over all $\ell \in\langle\varrho\rangle$, we are able to recover the erasures in $\mathcal{E}$ at the positions $\mathcal{R}$. Namely,

$$
\varepsilon_{\kappa_{\ell}, j_{\ell}}=e_{j_{\ell}}^{(\ell)} \beta_{\kappa_{\ell}, j_{\ell}}^{1-\varrho}, \quad \ell \in\langle\varrho\rangle .
$$

This, in turn, allows us to eliminate the symbol erasures from E.

Fig. 3 summarizes the decoding algorithm of a combination of errors of type (T1), (T2), and (T4). The complexity of Step 1 is $O((d+m) m n)$ operations in $F$ (see the discussion that precedes Example 11). Step 2 requires $O(d \rho \varrho)$ operations. Each iteration in Step 3 requires $O(d \varrho)$ operations (for Step 3a), $O\left(d^{2}\right)$ operations (for Step $3 \mathrm{~b}$ ), and $O(d m)$ operations (for Step 3c), totaling to $O(d(d+m) \varrho)$ for Step 3. Step 4 requires $O\left(d^{2} m\right)$ operations to compute the error-locator and errorevaluator polynomials, and an additional $O(d n)$ for the Chien search. Finally, Step 5 requires $O\left(d m^{2}\right)$ operations. To summarize, the decoding complexity amounts to $O((d+m) m n)$ operations for syndrome computation, $O(d n)$ for the Chien search, and $O(d(d+m) m)$ for the remaining steps.

C. Decoding of Errors and Erasures of Type (T1), (T2), (T4), and with Restrictions on Errors of Type (T3)

In this section, we consider the decoding of $\mathbb{C}$ when constructed as in Example 11, under certain assumptions on the set $\mathcal{L}$, namely, under some restrictions on the symbol error positions (errors of type (T3)). These restrictions always hold when $|\mathcal{L}| \leq 3$ and $d$ is sufficiently large.

Specifically, we consider the case where each column, except possibly for one column, contains at most one symbol error. The general strategy will be to locate the positions of these errors, thereby reducing to the case considered in Section IV-B We use the same notation as in that section, except that the set $\mathcal{L}$ is not necessarily empty and that (for reasons of simplicity) the set $\mathcal{R}$ is empty. As in Section IV-B, the number $\tau$ of block errors and the number $\rho$ of block erasures satisfy $2 \tau+\rho \leq d-2$. 


\section{Input:}

- Array $\Upsilon$ of size $m \times n$ over $F$.

- Set $\mathcal{K}$ of indexes of column erasures.

- Set $\mathcal{R}=\left\{\left(\kappa_{\ell}, j_{\ell}\right)\right\}_{\ell \in\langle\varrho\rangle}$ of positions of symbol erasures.

Steps:

1) Compute the $m \times(d-1)$ syndrome array

$$
S=\left(H_{0} \Upsilon_{0}\left|H_{1} \Upsilon_{1}\right| \ldots \mid H_{n-1} \Upsilon_{n-1}\right) H_{\text {GRS }}^{\top} .
$$

2) Compute the modified syndrome array to be the unique $\varrho \times$ $(d-1)$ matrix $\sigma$ that satisfies the congruence

$$
\sigma(y, x) \equiv S(y, x) \prod_{j \in \mathcal{K}}\left(1-\alpha_{j} x\right) \quad\left(\bmod \left\{x^{d-1}, y^{\varrho}\right\}\right) .
$$

3) For every $\ell \in\langle\varrho\rangle$ do:

a) Compute row $\varrho-1$ in the unique $\varrho \times(d-1)$ matrix $\sigma^{(\ell)}$ that satisfies the congruence

$$
\begin{array}{r}
\sigma^{(\ell)}(y, x) \equiv \mathrm{B}^{(\ell)}(y) \sigma(y, x)\left(1-\alpha_{j_{\ell}} x\right) \\
\left(\bmod \left\{x^{d-1}, y^{\varrho}\right\}\right),
\end{array}
$$

where $\mathrm{B}^{(\ell)}(y)$ is as in 27.

b) Decode $e_{j_{\ell}}^{(\ell)}$ (i.e., entry $j_{\ell}$ in $\boldsymbol{e}^{(\ell)}$ ) by applying a decoder for $\mathcal{C}_{\text {GRS }}$ using row $\varrho-1$ in $\sigma^{(\ell)}$ as syndrome and assuming that columns indexed by $\mathcal{K} \cup\left\{j_{\ell}\right\}$ are erased. Compute $\varepsilon_{\kappa_{\ell}, j_{\ell}}=e_{j \ell}^{(\ell)} \cdot \beta_{\kappa_{\ell}, j_{\ell}}^{1-\varrho}$.

c) Update the received array $\Upsilon$ and the syndrome array $S$ by

$$
\begin{aligned}
& \Upsilon(y, x) \leftarrow \Upsilon(y, x)-\varepsilon_{\kappa_{\ell}, j_{\ell}} \cdot x^{j_{\ell}} y^{\kappa_{\ell}} \\
& S(y, x) \leftarrow S(y, x)-\varepsilon_{\kappa_{\ell}, j_{\ell}} \cdot \mathrm{T}_{d-1}\left(x ; \alpha_{j_{\ell}}\right) \cdot \mathrm{T}_{m}\left(y ; \beta_{j_{\ell}}\right) .
\end{aligned}
$$

4) For every $h \in\langle m\rangle$, apply a decoder for $\mathcal{C}_{\text {GRS }}$ using row $h$ of $S$ as syndrome and assuming that columns indexed by $\mathcal{K}$ are erased. Let $E$ be the $m \times n$ matrix whose rows are the decoded error vectors for all $h \in\langle m\rangle$.

5) Compute the error array

$$
\mathcal{E}=\left(H_{0}^{-1} E_{0}\left|H_{1}^{-1} E_{1}\right| \ldots \mid H_{n-1}^{-1} E_{n-1}\right) .
$$

\section{Output:}

- Decoded array $\Upsilon-\mathcal{E}$ of size $m \times n$.

Fig. 3. Decoding of errors and erasures of type (T1), (T2), (T4), but not of type (T3). (See Section IV-B)

When $\vartheta=|\mathcal{L}|>0$, we write $\mathcal{L}=\left\{\left(\kappa_{\ell}, j_{\ell}\right)\right\}_{\ell \in\langle\vartheta\rangle}$, and assume that that there exists a $w \in\langle\vartheta\rangle$ such that the values $j_{0}, j_{1}, \ldots, j_{w}$ are all distinct, while $j_{w}=j_{w+1}=\cdots=j_{\vartheta-1}$. Furthermore, $\vartheta$ and $w$ should satisfy the inequalities

$$
\begin{aligned}
\vartheta & \leq \frac{m}{2}, \\
w+\tau+\rho & \leq d-2 .
\end{aligned}
$$

(While the inequality in (30) is already part of the requirements in Theorem 5 we need the inequality in 31 so that (13) will hold. Specifically, the inequality in 31 says that the number of erroneous columns does not exceed $d-1$. Observe that the inequality $2 \tau+\rho \leq d-2$ and the inequality in 30 together imply (31) whenever $m \leq d-\rho$.)

Without any loss of generality, we will also assume that $\varepsilon_{\kappa_{\ell}, j_{\ell}} \neq 0$ for every $\ell \in\langle\vartheta\rangle$. The set $\left\{j_{\ell}\right\}_{\ell \in\langle w+1\rangle}$ will be denoted hereafter by $\mathcal{L}^{\prime}$. When $\vartheta=0$, we formally define $w$ to be 0 and $\mathcal{L}^{\prime}$ to be the empty set.

Let the modified syndrome $\sigma$ be the unique $m \times(d-1)$ matrix that satisfies

$$
\sigma(y, x) \equiv S(y, x) \cdot \prod_{j \in \mathcal{K}}\left(1-\alpha_{j} x\right) \quad\left(\bmod x^{d-1}\right),
$$

and let $\tilde{S}$ be the $m \times(d-1-\rho)$ matrix formed by the columns of $\sigma$ that are indexed by $\langle\rho, d-1\rangle$. We recall from (13) that $\mu=\operatorname{rank}(\tilde{S})=\operatorname{rank}\left((E) \mathcal{J} \cup \mathcal{L}^{\prime}\right)$.

If $\mu \geq 2 w+2$, then we can regard the columns that are indexed by $\mathcal{L}^{\prime}$ as full block errors (namely, errors of type (T1)), and the conditions of Lemma 3 will still be satisfied, namely, we will have

$$
2(\tau+w+1)+\rho \leq d+\mu-2 .
$$

Therefore, we assume from now on that $\mu \leq 2 w+1$.

By 13 we get that for every $j \in \mathcal{J} \cup \mathcal{L}^{\prime}$, column $E_{j}$ belongs to colspan $(\tilde{S})$. In particular, this holds for $j \in \mathcal{L}^{\prime} \backslash\left\{j_{w}\right\}$, in which case $E_{j}$ (in polynomial notation) takes the form

$$
E_{j}(y)=\varepsilon_{\kappa, j} \cdot \mathrm{T}_{m}\left(y ; \beta_{\kappa, j}\right) .
$$

Let the row vectors $\boldsymbol{a}_{0}, \boldsymbol{a}_{1}, \ldots, \boldsymbol{a}_{m-\mu-1}$ form a basis of the dual space of $\operatorname{colspan}(\tilde{S})$, and for every $i \in\langle m-\mu\rangle$, let $a_{i}(y)$ denote the polynomial of degree less than $m$ with coefficient vector $\boldsymbol{a}_{i}$; we can further assume that this basis is in echelon form, i.e., $\operatorname{deg} a_{0}(y)<\operatorname{deg} a_{1}(y)<\ldots<$ $\operatorname{deg} a_{m-\mu-1}(y)<m$. This, in turn, implies that the degree of $a(y)=\operatorname{gcd}\left(a_{0}(y), a_{1}(y), \ldots, a_{m-\mu-1}(y)\right)$ satisfies

$$
\operatorname{deg} a(y) \leq \mu,
$$

namely, $a(y)$ has at most $\mu(\leq 2 w+1)$ distinct roots in $F$. Now, it is easy to see that for every $\xi \in F$, the column vector $\left(\xi^{h}\right)_{h \in\langle m\rangle}$ (also represented as $\mathrm{T}_{m}(y ; \xi)$ ) belongs to

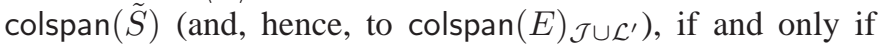
$\xi$ is a root of $a(y)$. In particular, $\beta_{\kappa_{\ell}, j_{\ell}}$ is a root of $a(y)$ for every $\ell \in\langle w\rangle$. We denote by $\mathcal{R}$ the root subset

$$
\mathcal{R}=\left\{(\kappa, j): a\left(\beta_{\kappa, j}\right)=0\right\},
$$

and define the polynomial $\mathrm{A}(y)$ by

$$
\mathrm{A}(y)=\sum_{i=0}^{\eta} \mathrm{A}_{i} y^{i}=\prod_{(\kappa, j) \in \mathcal{R}}\left(1-\beta_{\kappa, j} y\right)
$$

where $\eta=|\mathcal{R}|$.

Consider the $(m-\eta) \times n$ matrix $\hat{E}=\left(\hat{e}_{h, j}\right)_{h \in\langle m-\eta\rangle, j \in\langle n\rangle}$ which is formed by the rows of $\mathrm{A}(y) E(y, x)$ that are indexed by $\langle\eta, m\rangle$. Specifically,

$$
\hat{e}_{h, j}=\sum_{i=0}^{\eta} \mathrm{A}_{i} e_{h+\eta-i, j}, \quad h \in\langle m-\eta\rangle, \quad j \in\langle n\rangle
$$

(compare with 29). Respectively, let $\hat{S}$ be the $(m-\eta) \times$ $(d-1-\rho)$ matrix formed by the rows of $\mathrm{A}(y) \tilde{S}(y, x)$ that are indexed by $\langle\eta, m\rangle$. It readily follows that $\hat{E}_{j_{\ell}}(y)=0$ for $\ell \in\langle w\rangle$ and that

$\hat{E}_{j_{w}}(y)=\sum_{\ell \in\langle w, \vartheta\rangle}\left(\varepsilon_{\kappa_{\ell}, j_{w}} \beta_{\kappa_{\ell}, j_{w}}^{\eta} \mathrm{A}\left(\beta_{\kappa_{\ell}, j_{w}}^{-1}\right)\right) \cdot \mathrm{T}_{m-\eta}\left(y ; \beta_{\kappa_{\ell}, j_{w}}\right)$. 
Observe that the number of summands on the right-hand side of (34) is $\vartheta-w$, and that number is bounded from above by $m-2 w-1 \leq m-\mu \leq m-\eta$. This means that $\hat{E}_{j_{w}}(y)=0$ if and only if $\mathrm{A}\left(\beta_{\kappa_{\ell}, j_{w}}^{-1}\right)=0$ for all $\ell \in\langle w, \vartheta\rangle$. We also recall that

$$
\operatorname{rank}(\hat{S})=\operatorname{rank}\left((\hat{E})_{\mathcal{J} \cup\left\{j_{w}\right\}}\right)=\mu-\eta .
$$

Next, we distinguish between the following three cases.

Case 1: $\eta=\mu$. By 35 we must have $\hat{E}_{j_{w}}(y)=0$, which is equivalent to having $\mathrm{A}\left(\beta_{\kappa_{\ell}, j_{\ell}}^{-1}\right)=0$ for all $\ell \in\langle\vartheta\rangle$. Thus, assuming this case, we have $\mathcal{L} \subseteq \mathcal{R}$, and the decoding problem then reduces to the one discussed in Section IV-B.

Case 2: $\eta=\mu-1$. If $\hat{E}_{j_{w}}(y)=0$ then $\mathcal{L} \subseteq \mathcal{R}$. Otherwise, it follows from (35) that each column in $\hat{S}$ must be a scalar multiple of $\hat{E}_{j_{w}}$. The entries of $\hat{E}_{j_{w}}$, in turn, form a sequence that satisfies the (shortest) linear recurrence

$$
\mathrm{B}(y)=\sum_{i=0}^{\left|\mathcal{R}^{\prime}\right|} \mathrm{B}_{i} y^{i}=\prod_{(\kappa, j) \in \mathcal{R}^{\prime}}\left(1-\beta_{\kappa, j} y\right),
$$

where

$$
\mathcal{R}^{\prime}=\left\{\left(\kappa_{\ell}, j_{w}\right): \ell \in\langle w, \vartheta\rangle \text { and } \mathrm{A}\left(\beta_{\kappa_{\ell}, j_{w}}^{-1}\right) \neq 0\right\} .
$$

Indeed, this recurrence is uniquely determined, since the number of entries in $\hat{E}_{j_{w}}$, which is $m-\eta=m-\mu+1 \geq$ $m-2 w$, is at least twice the degree $\left|\mathcal{R}^{\prime}\right|(\leq \vartheta-w)$ of $\mathrm{B}(y)$. The recurrence can be computed efficiently from any nonzero column of $\tilde{S}$ using Massey's algorithm for finding the shortest linear feedback shift register capable of generating a prescribed finite sequence of symbols [23] (cf. BerlekampMassey algorithm as in, e.g., [26]). We now have $\mathcal{L} \subseteq \mathcal{R} \cup \mathcal{R}^{\prime}$, where

$$
\begin{aligned}
\left|\mathcal{R} \cup \mathcal{R}^{\prime}\right| & =|\mathcal{R}|+\left|\mathcal{R}^{\prime}\right| \\
& \leq \eta+\vartheta-w \\
& \leq 2 w+\vartheta-w \\
& =\vartheta+w \\
& <m .
\end{aligned}
$$

So the decoding problem again reduces to that in Section IV-B

Case 3: $\eta \leq \mu-2$. If $\hat{E}_{j_{w}}(y)=0$ then (again) $\mathcal{L} \subseteq \mathcal{R}$. Otherwise, the conditions of Lemma 3 hold with respect to $\hat{E}$ and to the matrix $\hat{Z}$ formed by the rows of $\mathrm{A}(y) Z(y, x)$ indexed by $\langle\eta, m\rangle$ (each such row is a codeword of $\mathcal{C}_{\mathrm{GRS}}$ ). Hence, we can decode $\hat{E}$. Next, we observe from (34) that for $j=j_{w}$, the vector $\hat{E}_{j}(y)$ can be seen as a syndrome of the column vector

$$
\mathcal{E}_{j}^{*}(y)=\sum_{\kappa \in\langle m\rangle: \mathrm{A}\left(\beta_{\kappa, j}^{-1}\right) \neq 0} \varepsilon_{\kappa, j} y^{\kappa}
$$

with respect to the following $(m-\eta) \times m$ parity-check matrix of a GRS code:

$$
H_{\mathrm{GRS}}^{(j)}=\left(v_{\kappa, j} \beta_{\kappa, j}^{h}\right)_{h \in\langle m-\eta\rangle, \kappa \in\langle m\rangle},
$$

where

$$
v_{\kappa, j}=\left\{\begin{array}{cl}
\beta_{\kappa, j}^{\eta} \mathrm{A}\left(\beta_{\kappa, j}^{-1}\right) & \text { if } \mathrm{A}\left(\beta_{\kappa, j}^{-1}\right) \neq 0 \\
1 & \text { otherwise }
\end{array} .\right.
$$

And since the Hamming weight of $\mathcal{E}_{j}^{*}$ is at most $\vartheta-w<$ $(m-\eta) / 2$, we can decode $\mathcal{E}_{j}^{*}$ uniquely from $\hat{E}_{j}$ (again, under the running assumption that $j=j_{w}$ ). Thus, for every $\kappa$ such that $\mathrm{A}\left(\beta_{\kappa, j}^{-1}\right) \neq 0$, we can recover the error value $\varepsilon_{\kappa, j}$ and subtract it from the respective entry of $\Upsilon$, thereby making $\mathcal{R}$ a superset of the remaining symbol errors. The problem though is that we do not know the index $j_{w}$. Therefore, we apply the above process to every nonzero column in $\hat{E}$ with index $j \notin \mathcal{K}$. A decoding failure means that $j$ is certainly not $j_{w}$, and a decoding success for $j \neq j_{w}$ will just cause us to incorrectly change already corrupted-columns in $\Upsilon$, without introducing new erroneous columns. We can then proceed with the decoding of $\Upsilon$ as in Section IV-B.

Fig. 4 presents the implied decoding algorithm of a combination of errors of type (T1), (T2), and (T3), provided that the type-(T3) errors satisfy the assumptions laid out at the beginning of this section; as said earlier, these assumptions hold when $m \leq d-\rho$ and the number of type-(T3) errors is at most 3. Steps 1,3, 6a, and 7, in Fig. 4 are essentially applications of steps in Figs. 2 and 3 . Next, we analyze the complexity of the remaining steps in Fig. 4, starting with Step 4a A basis in echelon form of the left kernel of $\tilde{S}$ can be found using $O\left(d^{2} m\right)$ operations in $F$, and from this basis we can compute $a(y)$ using $m$ applications of Euclid's algorithm, amounting to $O\left(d m^{2}\right)$ operations. The set $\mathcal{R}$ can then be found in Step $4 \mathrm{~b}$ via a Chien search, requiring $O(m n \cdot \min (d, m))$ operations, followed by $O\left(d^{2} m\right)$ operations to compute the matrix $\hat{S}$ in Step $4 \mathrm{c}$. The complexity of Step 5 is dictated by Step $5 b$ therein which, with a Chien search, can be implemented using $O\left(\mathrm{~m}^{2} n\right)$ operations. Finally, Step 6b requires $O(d(d+m) m)$ operations in $F$. In summary, the decoding complexity of the algorithm in Fig. 4 amounts to $O((d+m) m n)$ operations for syndrome computation and the Chien search, and $O(d(d+m) m)$ operations for the other steps.

\section{ACKNOWLEDGMENT}

The authors thank Erik Ordentlich for helpful discussions.

\section{APPENDIX A}

ANALYSIS FOR EXAMPLE 16

We derive the lower bound (22) on the expression (21):

$$
\begin{aligned}
\sum_{i=1}^{v}\left(\mathrm{r}_{i}-\mathrm{r}_{i-1}\right)\left(\mathrm{D}_{i-1}-1-2 \tau\right) \\
\quad \stackrel{1}{\geq} \sum_{i=1}^{v}\left(\mathrm{r}_{i}-\mathrm{r}_{i-1}\right)\left(\left\lceil\frac{2 \vartheta+1}{\mathrm{~d}_{i-1}}\right\rceil-1\right) \\
\geq \sum_{i=1}^{v}\left(\mathrm{r}_{i}-\mathrm{r}_{i-1}\right)\left(\left\lceil\frac{2 \vartheta+1}{\mathrm{r}_{i-1}+1}\right\rceil-1\right) \\
=\sum_{i=1}^{v} \sum_{j=\mathrm{r}_{i-1}+1}^{\mathrm{r}_{i}}\left(\left\lceil\frac{2 \vartheta+1}{\mathrm{r}_{i-1}+1}\right\rceil-1\right) \\
\geq \sum_{j=1}^{\mathrm{r}_{v}}\left(\left\lceil\frac{2 \vartheta+1}{j}\right\rceil-1\right)
\end{aligned}
$$




\section{Input:}

- Array $\Upsilon$ of size $m \times n$ over $F$.

- Set $\mathcal{K}$ of indexes of column erasures.

\section{Steps:}

1) Compute the $m \times(d-1)$ syndrome array

$$
S=\left(H_{0} \Upsilon_{0}\left|H_{1} \Upsilon_{1}\right| \ldots \mid H_{n-1} \Upsilon_{n-1}\right) H_{\mathrm{GRS}}^{\top} .
$$

2) Compute the $m \times(d-1-\rho)$ matrix $\tilde{S}$ formed by the columns of $S(y, x) \prod_{j \in \mathcal{K}}\left(1-\alpha_{j} x\right)$ that are indexed by $\langle\rho, d-1\rangle$. Let $\mu=\operatorname{rank}(\tilde{S})$.

3) (Attempt to correct assuming $\left|\mathcal{L}^{\prime}\right| \leq \mu / 2$.) Apply Steps 3 4 in Fig. 2 (with $K=\mathcal{K}$ ) to the modified syndrome array $\sigma(y, x)$, to produce an error array $E$. If decoding is successful, go to Step 8

4) a) Compute the greatest common divisor $a(y)$ of a basis of the left kernel of $\tilde{S}$.

b) Compute the set $\mathcal{R}$ and the polynomial $\mathrm{A}(y)$ as in 32 33. Let $\eta=|\mathcal{R}|$.

c) Compute the $(m-\eta) \times(d-1-\rho)$ matrix $\hat{S}$ formed by the rows of $\mathrm{A}(y) \tilde{S}(y, x)$ that are indexed by $\langle\eta, m\rangle$.

5) If $\eta=\mu-1$ then do:

a) Compute the shortest linear recurrence $\mathrm{B}(y)$ of any nonzero column in $\hat{S}$.

b) Compute the set

$$
\mathcal{R}^{\prime}=\left\{(\kappa, j): \mathrm{A}\left(\beta_{\kappa, j}^{-1}\right) \neq 0 \text { and } \mathrm{B}\left(\beta_{\kappa, j}^{-1}\right)=0\right\} .
$$

c) If $\left|\mathcal{R}^{\prime}\right|=\operatorname{deg} \mathrm{B}(y)$ and $\left|\mathcal{R}^{\prime}\right| \leq m-\eta$ then update $\mathcal{R} \leftarrow \mathcal{R} \cup \mathcal{R}^{\prime}$.

6) Else if $\eta \leq \mu-2$ then do:

a) Apply Steps 24 in Fig. 2 (with $K=\mathcal{K}$ ) to the syndrome array $\hat{S}$, to produce an error array $\hat{E}$.

b) For every index $j \notin \mathcal{K}$ of a nonzero column of $\hat{E}$ do:

i) Apply a decoder for the GRS code with the paritycheck matrix $H_{\mathrm{GRS}}^{(j)}$ as in 36-37, with $\hat{E}_{j}$ as syndrome, to produce an error vector $\mathcal{E}_{j}^{*}$.

ii) If decoding in Step 6(b)i is successful then let $E_{j}^{*}=$ $H_{j} \mathcal{E}_{j}^{*}$ and update $\Upsilon_{j} \leftarrow \Upsilon_{j}-\mathcal{E}_{j}^{*}$ and $S(y, x) \leftarrow$ $S(y, x)-E_{j}^{*}(y) \cdot \mathrm{T}_{d-1}\left(x ; \alpha_{j_{\ell}}\right)$.

7) Apply Steps 24 in Fig. 3 to $S, \mathcal{K}$, and $\mathcal{R}$, to produce an error array $E$.

8) Compute the error array

$$
\mathcal{E}=\left(H_{0}^{-1} E_{0}\left|H_{1}^{-1} E_{1}\right| \ldots \mid H_{n-1}^{-1} E_{n-1}\right) .
$$

\section{Output:}

- Decoded array $\Upsilon-\mathcal{E}$ of size $m \times n$.

Fig. 4. Decoding of errors and erasures of type (T1), (T2), (T4), and with restrictions on errors of type (T3). (See Section [V-C]) For the sake of simplicity, we assume that there are no erasures of type (T4).

$$
\begin{aligned}
& \geq \sum_{j=1}^{2 \vartheta}\left(\left\lceil\frac{2 \vartheta+1}{j}\right\rceil-1\right) \\
& =\sum_{j=1}^{\vartheta}\left\lceil\frac{2 \vartheta+1}{j}\right\rceil,
\end{aligned}
$$

where the penultimate step follows from $r_{v} \geq 2 \vartheta$. The lower bound (22) immediately follows by the known expression for harmonic sums [11, p. 264].

\section{REFERENCES}

[1] K.A.S. AbDel-GhafFar And M. HASSnER, Multilevel error-control codes for data storage channels, IEEE Trans. Inf. Theory, 37 (1991) 735741.

[2] M. Blaum, J.L. Hafner, and S. Hetzler, Partial-MDS codes and their application to RAID type of architectures, IBM Res. Rep. No. RJ10498 (February 2012).

[3] D. Bleichenbacher, A. Kiayas, and M. Yung, Decoding interleaved Reed-Solomon codes over noisy channels, Theor. Comput. Sci., 379 (2007), 348-360.

[4] E.L. BloKh AND V.V. Zyablov, Coding of generalized concatenated codes, Probl. Inf. Transm., 10 (1974), 218-222.

[5] A. Brown, L. Minder, And A. Shokrollahi, Probabilistic decoding of interleaved $R S$-codes on the $Q$-ary symmetric channel, Proc. IEEE Int. Symp. Inf. Theory, Chicago, IL, 2004, 326.

[6] D. Coppers mith AND M. Sudan, Reconstructing curves in three (and higher) dimensional space from noisy data, Proc. 35th Annual ACM Symp. Theory of Computing, San Diego, CA, 2003, 136-142.

[7] I. Dumer, Concatenated codes and their multilevel generalizations, in Handbook of Coding Theory, Volume II, V.S. Pless, W.C. Huffman (Editors), North-Holland, Amsterdam, 1998, 1911-1988.

[8] G.-L. Feng And K.K. Tzeng, A generalized Euclidean algorithm for multisequence shift-register synthesis, IEEE Trans. Inf. Theory, 35 (1989), 584-594.

[9] G.-L. Feng And K.K. Tzeng, A generalization of the BerlekampMassey algorithm for multisequence shift-register synthesis with applications to decoding cyclic codes, IEEE Trans. Inf. Theory, 37 (1991), $1274-1287$.

[10] R. Gabrys, E. YAakobi, And L. DOLECEK, Graded bit errorcorrecting codes with applications to flash memory, IEEE Trans. Inf. Theory, 59 (2013), 2315-2327.

[11] R.L. Graham, D.E. Knuth, and O. Patashnik, Concrete Mathematics, Addison-Wesley, Reading, MA, 1989.

[12] V. GURUSWAMI AND M. SUDAN, Improved decoding of Reed-Solomon and algebraic-geometry codes, IEEE Trans. Inf. Theory, 45 (1999), $1757-1767$.

[13] V. Gurus WAmi And C. XING, List decoding Reed-Solomon, algebraicgeometric, and Gabidulin subcodes up to the Singleton bound, Proc. 45th annual ACM Symp. on Theory of Computing, Palo Alto, California (June 2013), 843-852.

[14] C. Haslach AND A.J.H. VincK, A decoding algorithm with restrictions for array codes, IEEE Trans. Inf. Theory, 45 (1999), 2339-2344 (and correction in the same publication, 47 (2001), 479).

[15] C. Haslach and A.J.H. Vinck, Efficient decoding of interleaved linear block codes, Proc. IEEE Int. Symp. Inf. Theory, Sorrento, Italy, 2000, p. 149.

[16] J. Justesen, C. Thommesen, And T. Høholdt, Decoding of concatenated codes with interleaved outer codes, Proc. IEEE Int. Symp. Inform. Theory, Chicago, IL, 2004, p. 328.

[17] M. Kasahara, S. Hirasawa, Y. Sugiyama, and T. NameKawa, New classes of binary codes constructed on the basis of concatenated codes and product codes, IEEE Trans. Inf. Theory, 22 (1976), 462-467.

[18] R. KOETTER AND A. VARDY, The re-encoding transformation in algebraic list-decoding of Reed-Solomon codes, IEEE Trans. Inf. Theory, 57 (2011), 633-647.

[19] V.Yu. Krachkovsky, And Y.X. Lee, Decoding of parallel ReedSolomon codes with applications to product and concatenated codes, Proc. IEEE Int. Symp. Inf. Theory, Cambridge, MA, 1998, p. 55.

[20] H. Kurzweil, M. SEIDL, AND J.B. Huber, Reduced-complexity collaborative decoding of interleaved Reed-Solomon and Gabidulin codes, Proc. IEEE Int. Symp. Inf. Theory, St. Petersburg, Russia, 2011, 25572561.

[21] S. Lin And D.J. Costello, JR., Error Control Coding: Fundamentals and Applications, Prentice-Hall, Inc., Englewood Cliffs, New Jersey, 1983.

[22] F.J. MacWilliams and N.J.A. Sloane, The Theory of ErrorCorrecting Codes, North-Holland, Amsterdam, 1977.

[23] J.L. MASSEY, Shift-register synthesis and BCH decoding, IEEE Trans. Inf. Theory, 15 (1969), 122-127.

[24] J.J. Metzner and E.J. Kapturowski, A general decoding technique applicable to replicated file disagreement location and concatenated code decoding, IEEE Trans. Inf. Theory, 36 (1990), 1274-1287.

[25] W.W. Peterson And E.J. Weldon, JR., Error-Correcting Codes, MIT Press, Cambridge, MA, 1972.

[26] R.M. Roth, Introduction to Coding Theory, Cambridge Univ. Press, Cambridge, UK, 2006. 
[27] R.M. Roth AND G. Seroussi, Reduced-redundancy product codes for burst error correction, IEEE Trans. Inf. Theory, 44 (1998), 1395-1406.

[28] S. SAKATA, Decoding binary 2-D Cyclic codes by the 2-D BerlekampMassey algorithm, IEEE Trans. Inf. Theory, 37 (1991), 1200-1203.

[29] G. SCHMidT, V.R. Sidorenko, AND M. Bossert, Error and erasure correction of interleaved Reed-Solomon codes, in Coding and Cryptography, Ø. Ytrehus (Ed.), Lecture Notes in Computer Science, vol. 3969, Springer, Berlin (2006), 22-35.

[30] G. Schmidt, V.R. Sidorenko, And M. Bossert, Collaborative decoding of interleaved Reed-Solomon codes and concatenated code designs, IEEE Trans. Inf. Theory, 55 (2009), 2991-3011.

[31] V. Sidorenko And M. Bossert, Fast skew-feedback shift-register synthesis, Des., Codes and Crypt., (2012), 1-13.

[32] V. Sidorenko, G. Schmidt, AND M. Bossert, Decoding punctured Reed-Solomon codes up to the Singleton Bound, Proc. International ITG Conference on Source and Channel Coding, Erlangen, Germany, 2008, $1-6$.

[33] L. Storme And J.A. Thas, M.D.S. codes and $\operatorname{arcs}$ in $P G(n, q)$ with $q$ even: an improvement of the bounds of Bruen, Thas, and Blokhuis, $J$. Comb. Theory A, 62 (1993), 139-154.
[34] A. Wachter-Zeh, A. Zeh, AND M. Bossert, Decoding interleaved Reed-Solomon codes beyond their joint error-correcting capability, Des. Codes Cryptogr., to appear.

[35] L. WANG, Euclidean modules and multisequence synthesis, Lecture Notes in Computer Science, Ed. by S. Boztaş and I. E. Shparlinski, 2227 (2001), 239-248.

[36] J. Wu And D.J. Costello, JR., New multilevel codes over GF( $q$ ), IEEE Trans. Inf. Theory, 38 (1992), 933-939.

[37] Y. WU, New list decoding algorithms for Reed-Solomon and BCH codes, IEEE Trans. Inf. Theory, 54 (2008), 3611-3630.

[38] A. ZEH AND A. WACHTER, Fast multi-sequence shift-register synthesis with the Euclidean algorithm, Adv. Math. Comm., 5 (2011), 667-680.

[39] V.A. ZINOV'Ev, Generalized concatenated codes for channels with error bursts and independent errors, Probl. Inf. Transm., 17 (1981), 254-260.

[40] V.A. Zinov'ev AND V. Zyablov, Correction of error bursts and independent errors using generalized cascade codes, Probl. Inf. Transm., 15 (1979), 125-134. 